ARTICLE

https://doi.org/10.1038/s41467-019-10128-w

\title{
Genotype-covariate correlation and interaction disentangled by a whole-genome multivariate reaction norm model
}

Guiyan $\mathrm{Ni}^{1,2}$, Julius van der Werf ${ }^{2}$, Xuan Zhou ${ }^{1}$, Elina Hyppönen (i) ${ }^{1,3,4}$, Naomi R. Wray (i) ${ }^{5,6}$ \& S. Hong Lee ${ }^{1,2,6}$

The genomics era has brought useful tools to dissect the genetic architecture of complex traits. Here we propose a multivariate reaction norm model (MRNM) to tackle genotype-covariate (G-C) correlation and interaction problems. We apply MRNM to the UK Biobank data in analysis of body mass index using smoking quantity as a covariate, finding a highly significant G-C correlation, but only weak evidence for $\mathrm{G}-\mathrm{C}$ interaction. In contrast, G-C interaction estimates are inflated in existing methods. It is also notable that there is significant heterogeneity in the estimated residual variances (i.e., variances not attributable to factors in the model) across different covariate levels, i.e., residual-covariate (R-C) interaction. We also show that the residual variances estimated by standard additive models can be inflated in the presence of G-C and/or R-C interactions. We conclude that it is essential to correctly account for both interaction and correlation in complex trait analyses.

\footnotetext{
${ }^{1}$ Australian Centre for Precision Health, University of South Australia Cancer Research Institute, University of South Australia, Adelaide, South Australia 5000, Australia. ${ }^{2}$ School of Environmental and Rural Science, University of New England, Armidale, NSW 2351, Australia. ${ }^{3}$ Population, Policy and Practice, UCL Great Ormond Street Institute of Child Health, London WC1N 1EH, UK. ${ }^{4}$ South Australian Health and Medical Research Institute, Adelaide, South Australia 5000, Australia. ${ }^{5}$ Institute for Molecular Bioscience, University of Queensland, Brisbane, Queensland 4072, Australia. ${ }^{6}$ Queensland Brain Institute, University of Queensland, Brisbane, Queensland 4072, Australia. Correspondence and requests for materials should be addressed to S.H.L. (email: hong. lee@unisa.edu.au)
} 
$\mathrm{V}$ ariation in complex traits between people is determined both by genetic and non-genetic factors. The non-genetic component will include environmental risk factors, but also unknown factors that are characterised by stochastic variation. The interplay between genetic and identifiable environmental factors has long been a topic of research interest ${ }^{1-3}$, since the identification of genotype-environment interactions has the potential to inform on health interventions to overcome genetic predisposition to disease. However, many so-called environmental risk factors (e.g., smoking, alcohol consumption, stressful life events, educational attainment) are themselves complex traits whose variation also reflects both genetic and non-genetic factors. For example, the relationship between smoking and body mass index (BMI) is complex, i.e., common causal genetic variants have biological effects on both traits (pleiotropy or genetic correlation $)^{4}$ while BMI is also modulated by smoking status (interaction $)^{5-7}$ (Fig. 1). The relationship between smoking and BMI is a good example for a complex association which can be best modelled using a framework that can account both for genotype-covariate correlation and interaction (GCCI).

Both correlation ('association') and interaction ('effect modification') are fundamental in biology ${ }^{8-10}$, but it is critical to distinguish between them because their biological mechanisms differ, as do their implications. This association/interaction problem has been well posed in the classical twin study approach ${ }^{11}$, showing that association and interaction can be disentangled and correctly estimated with an appropriate model and sufficient data. Unfortunately, large well-powered data sets with measures on multiple family members are few. However, genome-wide association studies (GWAS) now provide different types of genetically informative data to investigate GCCI. The genomic era has brought useful tools to dissect the genetic architecture of complex traits, where genetic variance and covariance can be estimated based on genome-wide single nucleotide polymorphisms (SNPs) genotyped in large-scale population samples. The increased availability of sufficiently powered data sets, with information on measured genetic and non-genetic risk factors, motivates the need to develop appropriate statistical tools for GCCI analysis. Locus specific interaction at single locus level has been widely studied ${ }^{12}$.

\section{a Existing model}

Smoking

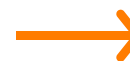

Genes

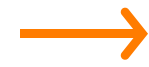

BMI

\section{b Proposed model}

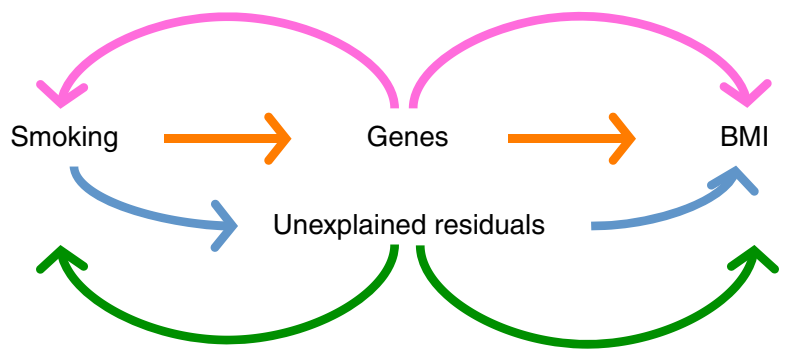

Fig. $1 \mathrm{~A}$ simplified path diagram of the existing and proposed interaction model. a Existing interaction model where smoking modulates genes such that their expression, regulation and effects underlying BMI are changed (genotype-covariate interaction). b Proposed MRNM where genotype-covariate interaction is only a part of the latent mechanism that also includes pleiotropic effects on smoking and BMI (magenta arrow), residual-covariate interaction (blue arrow) and residual correlation (green arrow). Unexplained residuals may be partly due to epigenetic or unknown factors that are not captured by the genetic component, resulting in heterogeneous error (co)variance across different covariate values
However, it is desirable to estimate whole-genome level interaction, which is accumulated from every locus across the genome and has a direct implication on actual phenotypic modulations.

The reaction norm model (RNM) has been developed and applied to genotype-environment interaction analyses in ecology $^{13}$ and agriculture ${ }^{14,15}$. The RNM allows environmental exposures to be modelled such that the genetic effects of a trait can be fitted as a nonlinear function of a continuous environmental gradient. The possible modulators of the phenotypes of the trait are not limited to environmental exposures, but can include any covariates, regulated by environmental and genetic factors, which are shared with the phenotypes. In other words, the genetic effect, and therefore the phenotype, of one trait often depends on the phenotype of another trait. This can be modelled by introducing dependence between the phenotype and the covariate, where the covariate represents the phenotype of the modulating trait, with both phenotypes having shared genetic and environmental components.

In the context of whole genome analyses of human complex traits, there is currently no approach that can fit GCCI effects to disentangle interaction from correlation at the genome-wide level. Yet, ignoring either the genotype-covariate $(\mathrm{G}-\mathrm{C})$ correlation or the $\mathrm{G}-\mathrm{C}$ interaction may cause biased estimates of variance components which form the basis of SNP-heritability or interaction estimation ${ }^{11}$. Random regression-genomic restricted maximum likelihood (RR-GREML) ${ }^{16,17}$ and G-C interaction (GCI)GREML $^{18}$ have been used to detect and estimate $\mathrm{G}-\mathrm{C}$ interaction at the whole genome level for BMI modulated by smoking quantity ${ }^{16}$. However, the analytical approach used in this study was based on univariate models which did not account for $\mathrm{G}-\mathrm{C}$ correlation, thereby assuming that there is no correlation between the covariates and the outcomes. This can inflate signals indicating the presence of $\mathrm{G}-\mathrm{C}$ interaction and lead to biased estimates by the failure to account for the $\mathrm{G}-\mathrm{C}$ correlation. A further limitation with the existing methods is that these cannot be applied to continuous covariates without an arbitrary stratification into discrete exposure groups ${ }^{16}$. Importantly, additive models used for the estimation of SNP heritability (such as GREML ${ }^{17,18}$ which is based on individual level data, or LDSC ${ }^{19-21}$ based on summary statistics) may give biased estimates for genetic and residual (error) variance if the trait of interest is moderated by (unknown) covariates due to failure in adequate capture of the interaction effects. It is currently not possible to use RR-GREML or GCI-GREML to assess such bias especially when using continuous covariates.

In this study, we develop a whole-genome reaction norm model (RNM) that is computationally flexible and powerful when estimating genome-wide $\mathrm{G}-\mathrm{C}$ interactions for complex traits. We also extend this approach to a whole-genome multivariate RNM (MRNM) framework to capture fully the GCCI effects, jointly modelling pleiotropy and interactions at the genome-wide level. As the proposed methods will be able to more realistically account for the complexity of GCCI effects, we hypothesise that they will lead to a significant reduction in bias and notably improve the estimation of the genetic architecture of complex traits.

\section{Results}

Overview of methods. We propose an extension of the wholegenome RNM that can estimate $\mathrm{G}-\mathrm{C}$ interactions, where covariates can be continuous phenotypes of traits correlated with the response. In a simplified form of this model, the response variable $(y)$ representing the main trait is modulated by a continuous covariate variable $(c)$ as

$$
y_{i}=g_{i}+e_{i}=\alpha_{i 0}+\alpha_{i 1} \cdot c_{i}+e_{i}
$$

where $g_{i}$ and $e_{i}$ are the genetic and residual effects for the $i^{\text {th }}$ 
individual record, and $g_{i}$ can be further decomposed to the zero and first order random regression coefficients, denoted as $\alpha_{i 0}$ and $\alpha_{i 1}$ (i.e., the regression coefficients may vary between individuals) and $c_{i}$ is the unique covariate value for the $i^{\text {th }}$ individual (see Methods for the formal model specification and covariance structure). This model is the same as RR-GREML ${ }^{16,17}$ proposed by Maier et al. ${ }^{22}$ except the fact that $c_{i}$ in our model is a continuous variable, as opposed to a discrete variable. The RNM can be generalised to account for residual heterogeneity or residual-covariate $(\mathrm{R}-\mathrm{C})$ interaction, by decomposing $e_{i}$ as follows:

$$
y_{i}=g_{i}+e_{i}=\alpha_{i 0}+\alpha_{i 1} \cdot c_{i}+\tau_{i 0}+\tau_{i 1} \cdot c_{i}
$$

where $\alpha_{i 0}, \alpha_{i 1}$, and $c_{i}$ are defined as above, and $\tau_{i 0}$ and $\tau_{i 1}$ are the zero and first order of random regression coefficients for the residual variance (see Methods).

The RNM described above is used to model $\mathrm{G}-\mathrm{C}$ and $\mathrm{R}-\mathrm{C}$ interaction effects without accounting for $\mathrm{G}-\mathrm{C}$ and $\mathrm{R}-\mathrm{C}$ correlation. As briefly explained in Introduction section, the same genetic factors can affect both the covariate trait and the main trait (response variable), and at the same time, the covariate trait phenotypes can directly modify the main trait. For example, both BMI and smoking have non-zero SNP-based heritability ${ }^{23}$, there is a direct genetic association between BMI and smoking quantity, and BMI is known to be modulated by smoking. Typically, the covariate itself (here, smoking) is affected by genetic effects and residual error (i.e., $c_{i}=\beta_{i}+\varepsilon_{i}$ ), and there can be non-negligible correlations between $\alpha_{0}$ and $\beta, \alpha_{1}$ and $\beta, \tau_{0}$ and $\varepsilon$, and $\tau_{1}$ and $\varepsilon$ (for the full covariance structure, see Methods). We used MRNM to take into account the $\mathrm{G}-\mathrm{C}$ and $\mathrm{R}-\mathrm{C}$ correlation.

We compared the performance of previously published methods (RR-GREML ${ }^{16,17}$ and GCI-GREML ${ }^{18}$ ), RNM, and MRNM using simulated and real data from the UK Biobank ${ }^{24}$ (see Methods and Supplementary Note 1). The models used in these comparisons are summarised in Supplementary Table 1. In the simulation, we used likelihood ratio tests to obtain the $p$ values, and assessed bias, type I error rate and power of detecting $\mathrm{G}-\mathrm{C}$ and $\mathrm{R}-\mathrm{C}$ interactions. In the analyses using the UK Biobank, we modelled BMI as the main trait and fitted separate models using information on pack years of smoking (SMK), neuroticism score (NEU) and the first principal component of genotypes (PC1) as the covariates. We used SMK and NEU because of their well-known association with $\mathrm{BMI}^{16,25,26}$ although the variance and covariance components of the interaction effects were not clearly known. We would expect to see little or no evidence for interaction due to $\mathrm{PC} 1$ because $\mathrm{BMI}$ was reported to have relatively small interaction across different populations ${ }^{27}$ and the data used in this study were stringently restricted according to their ancestry (see Methods). In addition, we applied the standard GREML $^{17,18}$ and LDSC ${ }^{19-21}$ methods to estimate SNP-based heritability for the main response variable $(y)$, where $y$ is modulated by one or more unknown covariates. With this analysis, we are able to explore the potential bias in results obtained by these methods in the presence of non-negligible interactions.

Type I error rate, power and estimates for G-C interaction. We used simulation (see Methods) to quantify type I error rate and power of detecting G-C interaction for the proposed RNM, RRGREML and GCI-GREML, without considering G-C correlation. As shown in Fig. 2, there was no inflation of type I error rate for all methods under the null model, when there was no G-C correlation and interaction. In contrast, when there were nonnegligible G-C interactions, RNM outperformed RR-GREML and GCI-GREML in detecting G-C interactions (Fig. 3). The power to detect G-C interaction was slightly higher for RR-GREML compared to GCI-GREML. The type I error rate and power of MRNM were very similar to those of RNM (Supplementary Fig. 1).

We also tested if the methods can give unbiased estimates for variance components of random regression coefficients underlying the mechanism of $\mathrm{G}-\mathrm{C}$ interaction. When $\mathrm{G}-\mathrm{C}$ interactions were present, RNM gave unbiased estimates, whereas estimates from RR-GREML and GCI-GREML differed from true values (Supplementary Table 2). Note that RR-GREML and GCIGREML required the stratification of the sample into discrete groups, resulting in an artificial heterogeneity of phenotypic variances across the discrete groups (Supplementary Fig. 2).

Type I error rate, power and estimates for GCCI. We also considered the GCCI model in simulations (Methods). Under the null (no G-C interaction), in the presence of non-negligible genetic correlations between the main response and covariate variables, we observed spurious signals for $\mathrm{G}-\mathrm{C}$ interaction in the univariate analysis using the RNM (Fig. 4). This was probably due to the fact that the unmodelled association of the main genetic effects between the phenotypes and covariate, $\operatorname{cov}\left(\boldsymbol{\alpha}_{0}, \boldsymbol{\beta}\right)$, was partly captured and estimated as interaction variance, $\operatorname{var}\left(\boldsymbol{\alpha}_{1} \cdot \mathbf{c}\right)$, from the model, $\mathbf{y}=$ $\boldsymbol{a}_{0}+\boldsymbol{a}_{1} \cdot \mathbf{c}+\mathbf{e}$. However, MRNM performed notably better in these analyses, being able to control for type I error rate (0.046) in detecting G-C interaction (Fig. 4). With a more modest genetic correlation (e.g., 0.1$)$, type I error rate was still inflated $(0.25$ in Supplementary Fig. 3) and the estimates were biased (Supplementary Table 3) when applying the univariate model.

In the presence of $\mathrm{G}-\mathrm{C}$ correlations and $\mathrm{G}-\mathrm{C}$ interactions, both RNM and MRNM performed similarly in detecting G-C interactions (Supplementary Fig. 4) although the significance of the $\mathrm{G}-\mathrm{C}$ interaction for RNM was slightly inflated due to overestimated parameters (see $\operatorname{var}\left(\boldsymbol{\alpha}_{1}\right)$ for RNM in Supplementary Table 4). Importantly, MRNM gave unbiased estimates for both G-C correlation and G-C interaction (Supplementary Table 4).

When using RNM, the spurious signals for detecting $\mathrm{G}-\mathrm{C}$ interaction could be controlled by adjusting the main response for the covariate, i.e., using residuals (as the response) from the regression of the main response on the covariate (Supplementary Figs. 5 and 6). However, such adjustment was crude, and the genuine effects were sometimes over-corrected, again leading to biased estimates especially in the estimated variance of the main effects (Supplementary Table 5).

Residual-covariate (R-C) correlation and interaction. In addition to GCCI, it is possible that the residual effects $\left(e_{i}\right)$ are correlated with the covariate $\left(c_{i}\right)$ and that there is interaction (RCCI) (see Eq. (3) or Methods). We tested various scenarios for detecting $\mathrm{G}-\mathrm{C}$ interactions in the presence of $\mathrm{R}-\mathrm{C}$ correlation and/or interaction (Supplementary Figs. 7-11). In the absence of $\mathrm{G}-\mathrm{C}$ interactions but with $\mathrm{R}-\mathrm{C}$ interactions, type I error rate was well controlled in all methods (Supplementary Fig. 7 and Supplementary Data 1). In the presence of $\mathrm{G}-\mathrm{C}$ interactions and $\mathrm{R}-\mathrm{C}$ interactions, RNM had greater power to detect $\mathrm{G}-\mathrm{C}$ interaction compared to RR-GREML or GCI-GREML (Supplementary Fig. 8 and Supplementary Data 2). In the absence of G-C interaction but with G-C correlations and RCCI, all three methods were able to control type I errors in detecting G-C interaction (Supplementary Fig. 9 and Supplementary Data 3). With the full GCCI model in the presence of $\mathrm{G}-\mathrm{C}$ correlation and interaction, and $\mathrm{R}-\mathrm{C}$ correlation and interaction, MRNM had greater power than RR-GREML or GCI-GREML (Supplementary Fig. 10 and Supplementary Data 4). When increasing the variance explained by the $\mathrm{G}-\mathrm{C}$ interaction, the statistical power reached $100 \%$ with all 

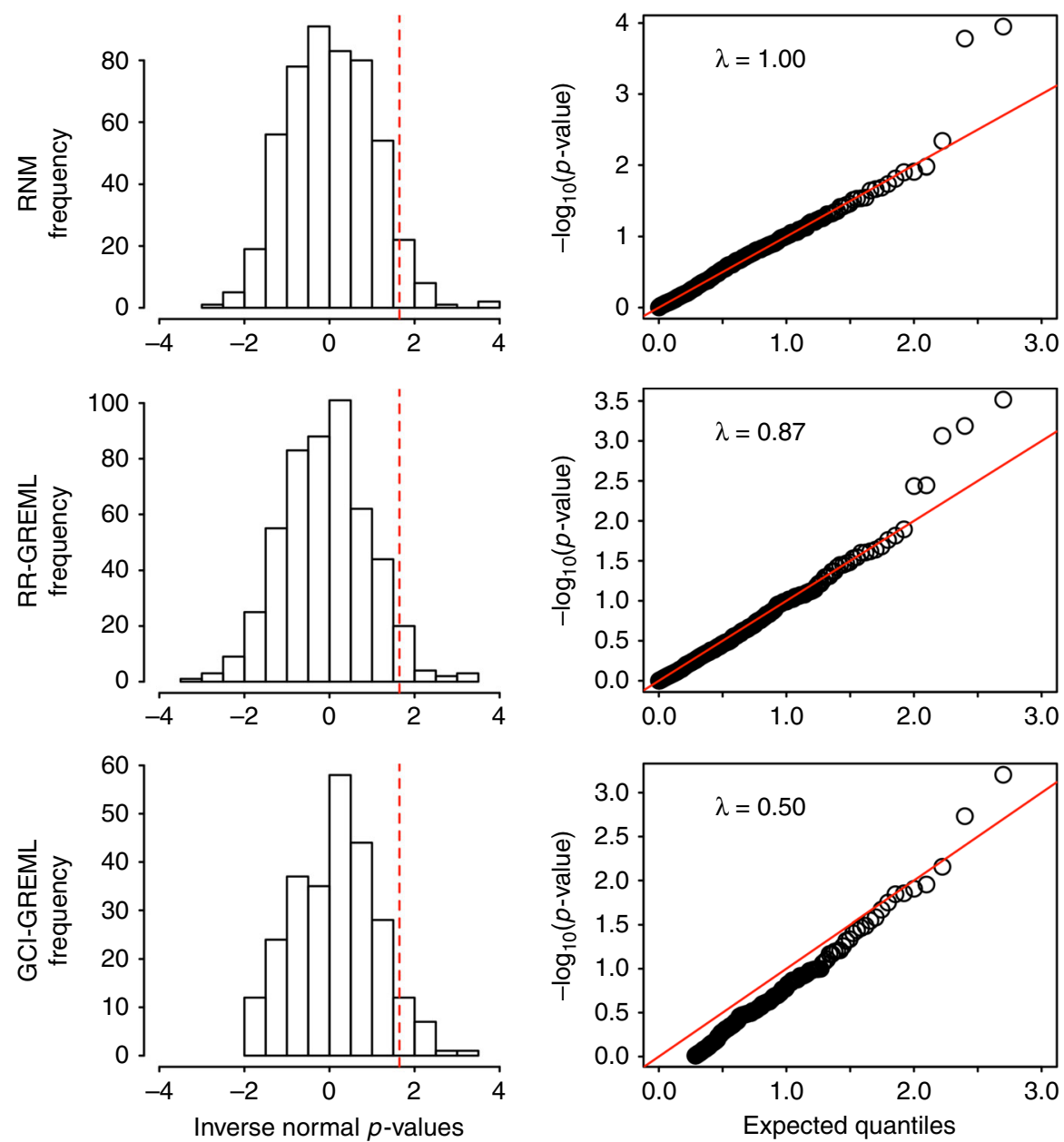

Fig. 2 Type I error rate for detecting G-C interaction is under control. Five hundred replicates of data were simulated under a null model that assumed no genotype-covariate interaction. Simulation was based QCed ARIC data consisting 7,263 individuals and 583,058 SNPs. The model is specified as $\mathbf{y}=$ $\boldsymbol{\alpha}_{\mathbf{0}}+\boldsymbol{\alpha}_{\mathbf{1}} \times \mathbf{c}+\mathbf{e}$ with $\mathbf{c}=\boldsymbol{\beta}+\boldsymbol{\varepsilon}$, all effects drawn from a multivariate normal distribution, where the variance-covariance structure between $\boldsymbol{\alpha}_{\mathbf{0}}, \boldsymbol{\beta}$, and $\boldsymbol{\alpha}_{\mathbf{1}}$ (in this order) is $\left[\begin{array}{lll}1 & 0 & 0 \\ 0 & 1 & 0 \\ 0 & 0 & 0\end{array}\right]$ and that between $\mathbf{e}$ and $\boldsymbol{\varepsilon}$ is $\left[\begin{array}{ll}1 & 0 \\ 0 & 1\end{array}\right]$. For every replicate, each of the three models was fitted to obtain a $p$-value for the $\mathrm{G}-\mathrm{C}$ interaction via a comparison between the null $\left(\mathrm{H}_{0}\right)$ and alternative hypothesis $\left(\mathrm{H}_{1}\right)$ models using a likelihood ratio test. For RNM, the $\mathrm{H}_{0}$ and $\mathrm{H}_{1}$ models were $\mathbf{y}=\boldsymbol{\alpha}_{\mathbf{0}}+\mathbf{e}$ and $\mathbf{y}=\boldsymbol{\alpha}_{\mathbf{0}}+\boldsymbol{\alpha}_{\mathbf{1}} \times \mathbf{c}+\mathbf{e}$. For RR-GREML and GCI-GREML, the $\mathrm{H}_{\mathrm{O}}$ and $\mathrm{H}_{1}$ models were $\mathbf{y}=\boldsymbol{\alpha}_{\mathbf{o}}+\mathbf{e}$ and $\mathbf{y}=\boldsymbol{\alpha}_{\mathbf{0}}+\boldsymbol{\alpha}_{\mathbf{1}} \times \mathbf{c}+\mathbf{e}$. In RR-GREML and GCI-GREML, samples were arbitrarily stratified into four different groups according to the covariate levels. RR-GREML explicitly estimate residual variance for each of the four groups whereas GCI-GREML assumes homogeneous residual variance across the four groups and estimates a single residual variance. The left panels show the proportions of significant $p$-values, i.e., type I error rate, for RNM, RR-GREML and GCI-GREML, which are 0.048 , 0.048 and 0.034 , respectively. Note that $p$-values are inverse normal transformed, such that the statistical significance level, i.e., 1.65 , shown as dashed lines, is equivalent to the 0.05 level before the transformation. The right panels are quantile-quantile (Q-Q) plots of $-\log _{10}(p$-values) from RNM, RR-GREML and GCI-GREML to detect G-C interaction using simulated data ( $\lambda$ is the ratio of observed to expected median test statistic). The low lambda values for RRGREML and GCl-GREML was probably due to the fact that the methods stratify individuals into multiple groups. In addition, GCI-GREML constrains the negative estimated variance to zero

three methods (Supplementary Fig. 11 and Supplementary Data 5). It is notable that MRNM gave unbiased estimates of the components whereas the other methods generated some degree of bias in the estimation (Supplementary Data 3-5, and Supplementary Table 6).

Inflated residual variance using LDSC or GREML. LDSC SNPbased heritability estimates have been widely used ${ }^{19,20,28}$. However, it is not clear if G-C or R-C interactions have an effect on LDSC SNP-based heritability estimations. When using LDSC, GWAS summary stats are typically used without knowing (accessing) the information of specific covariates. So, it is important to assess the biasness of estimates if interaction effects are not properly modelled either because of the model limitation (i.e., LDSC is an additive model) or lack of covariate information. With simulated data based on the G-C or R-C interaction model, we showed that both the GREML and LDSC overestimated the residual variance for the main response variable hence underestimating the SNP-based heritability (Fig. 5 and Supplementary Fig. 12). When the interaction component explained $10 \%$ of the total variance, the estimated residual variance based on GREML or LDSC was 1.5 times higher than the true simulated value (Fig. 5). When the variance of the interaction was increased to $25 \%$ of the total variance, GREML or LDSC overestimated the residual variance up to 3 -fold. However, RNM generated unbiased estimates for the residual variance in most cases. It was noted that the estimated genetic variance and covariance were 

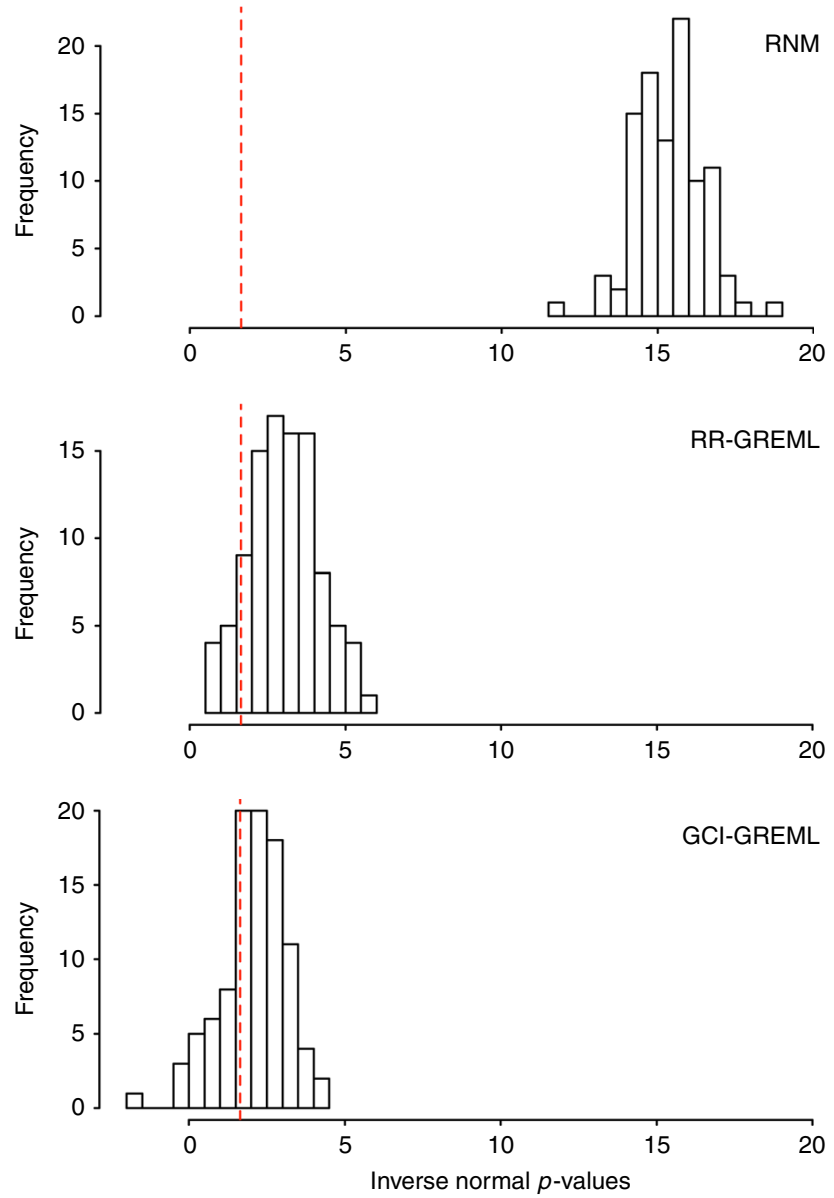

Fig. 3 RNM has more statistical power than RR-GREML and GCI-GREML. One hundred replicates of data were simulated under a model that assumed the presence of a genotype-covariate interaction. Simulation was based QCed ARIC data consisting 7,263 individuals and 583,058 SNPs. The model is specified as $\mathbf{y}=\boldsymbol{\alpha}_{\mathbf{0}}+\boldsymbol{\alpha}_{\mathbf{1}} \times \mathbf{c}+\mathbf{e}$ with $\mathbf{c}=\boldsymbol{\beta}+\boldsymbol{\varepsilon}$, all effects drawn from a multivariate normal distribution, where the variance-covariance structure between $\boldsymbol{\alpha}_{\mathbf{0}}, \boldsymbol{\beta}$, and $\boldsymbol{\alpha}_{\mathbf{1}}$ (in this order) is $\left[\begin{array}{ccc}1 & 0 & 0.05 \\ 0 & 1 & 0 \\ 0.05 & 0 & 0.25\end{array}\right]$ and that between $\mathbf{e}$ and $\boldsymbol{\varepsilon}$ is $\left[\begin{array}{ll}1 & 0 \\ 0 & 1\end{array}\right]$. For every replicate, each of the three models was fitted to obtain a $p$-value for the $\mathrm{G}-\mathrm{C}$ interaction via a comparison between the null $\left(\mathrm{H}_{0}\right)$ and alternative hypothesis $\left(\mathrm{H}_{1}\right)$ models using a likelihood ratio test. For RNM, the $\mathrm{H}_{0}$ and $\mathrm{H}_{1}$ models were $\mathbf{y}=\boldsymbol{\alpha}_{\mathbf{0}}+\mathbf{e}$ and $\mathbf{y}=\boldsymbol{\alpha}_{\mathbf{0}}+\boldsymbol{\alpha}_{\mathbf{1}} \times \mathbf{c}+\mathbf{e}$. For RR-GREML and GCI-GREML, the $\mathrm{H}_{\mathrm{O}}$ and $\mathrm{H}_{1}$ models were $\mathbf{y}=\boldsymbol{\alpha}_{\mathbf{0}}+\mathbf{e}$ and $\mathbf{y}=\boldsymbol{\alpha}_{\mathbf{0}}+\boldsymbol{\alpha}_{\mathbf{1}} \times \mathbf{c}+\mathbf{e}$. In RR-GREML and GCI-GREML, samples were arbitrarily stratified into four different groups according to the covariate levels. RR-GREML explicitly estimate residual variance for each of the four groups whereas GCl-GREML assumes homogeneous residual variance across the four groups and estimates a single residual variance. This figure shows the proportions of significant $p$-values, i.e., statistical power, for RNM, RR-GREML and GCI-GREML, which are 1, 0.9 and 0.69, respectively. Note that $p$-values are inverse normal transformed, such that the statistical significance level, i.e., 1.65, shown as dashed lines, is equivalent to the 0.05 level before the transformation

mostly unbiased whether using GREML, LDSC or RNM (Fig. 5 and Supplementary Table 7). This implies that a consistent estimate of SNP-based heritability can be obtained across the methods given that the phenotypic variance is correctly estimated on the original scale, which is invariant to whether there are interactions or not.
GCCI and RCCI analysis for real data (UK Biobank). We used the first release of UK Biobank (see Methods) to compare various models that test interaction using RR-GREML (M1) and GCIGREML (M2), and the proposed approaches RNM (M3-M7) and MRNM (M8-M12) (Table 1). We applied the models with BMI as the outcome trait using either SMK, NEU or PC1 as the covariate of interest, in order to detect $\mathrm{G}-\mathrm{C}$ and/or R-C interactions.

Table 1 shows the $p$-values for interaction effects from the likelihood ratio tests and the corresponding estimates for variance and covariance components are presented in Supplementary Data 6. We found that BMI was significantly modulated by SMK using RR-GREML (M1, $p$-value $=1.00 \mathrm{E}-03)$ or GCI-GREML $(\mathrm{M} 2, p$-value $=1.99 \mathrm{E}-07)$, confirming published results ${ }^{16}$. However, published methods did not account for G-C correlation or RCCI (Supplementary Data 4 and 5). Using RNM (M3-M7), we found that the combined $\mathrm{G}-\mathrm{C}$ and $\mathrm{R}-\mathrm{C}$ interaction effects were highly significant (M3-M5). We then used RNM to test for the G-C interaction corrected for R-C interaction (M7) and found similar results $\left(p\right.$-value $=1.83 \mathrm{E}-04$ and $\operatorname{var}\left(\boldsymbol{\alpha}_{1}\right)=0.47$ with $\mathrm{SE}=$ 0.12 ) compared to those obtained using RR-GREML (M1) and GCI-GREML (M2). It is noted that residual heterogeneity (reflected by $\mathrm{R}-\mathrm{C}$ interaction) was partly controlled in M1 and M2 as these models adjusted for group differences with the covariate stratified into four discrete groups, which however generated biased estimates as shown in Supplementary Data 4 and 5 from simulation. We next applied MRNM to test for interactions, accounting for both $\mathrm{G}-\mathrm{C}$ interaction and $\mathrm{G}-\mathrm{C}$ correlation effects (M8-M12). We found that the signal for the combined $\mathrm{G}-\mathrm{C}$ and $\mathrm{R}-\mathrm{C}$ interaction increased (M8-M10) compared to that seen using RNM, which turned out to be mostly due to the increased $\mathrm{R}-\mathrm{C}$ interaction (M11). It is likely that this was due to the large negative residual correlation between BMI and SMK (Fig. 6 and Supplementary Data 6) which could be more properly modelled in MRNM than in RNM. We finally tested $\mathrm{G}-\mathrm{C}$ interaction controlled for $\mathrm{G}-\mathrm{C}$ correlation, and $\mathrm{R}-\mathrm{C}$ correlation and $\mathrm{R}-\mathrm{C}$ interaction (M12), and showed that the signal for $\mathrm{G}-\mathrm{C}$ interaction was marginally significant ( $p$-value $=$ $3.26 \mathrm{E}-02)$. This was probably due to the fact that the nonnegligible G-C correlation (Fig. 6 and Supplementary Data 6) would inflate the signal of G-C interaction in M1, 2 and 7 (all based on univariate framework). As shown in the simulations, the MRNM was the most reliable model (Supplementary Data 4 and 5). Hence, this demonstrates that conclusions from models using the MRNM applied to real data can differ from those obtained using methods based on more simplified models (Fig. 6 and Supplementary Data 6).

We also analysed BMI using $\mathrm{NEU}^{25,26}$ as the covariate in the various models, observing evidence for interaction with RRGREML ( $p$-value $=6.82 \mathrm{E}-04)$ but not GCI-GREML(M1 and M2 in Table 1). We found strong $\mathrm{G}-\mathrm{C}$ and $\mathrm{R}-\mathrm{C}$ interactions when using either RNM (M3-M5) or MRNM (M8-M10). Evidence for interaction remained when the $\mathrm{G}-\mathrm{C}$ interaction effects were adjusted for $\mathrm{R}-\mathrm{C}$ interaction effects $(p$-value $=3.77 \mathrm{E}$ 04 for M7 and 1.08E-03 for M12) or vice versa (7.73E-06 for M6 and $2.36 \mathrm{E}-05$ for M11). This shows that $\mathrm{G}-\mathrm{C}$ and $\mathrm{R}-\mathrm{C}$ interactions are both important and contribute to the shared aetiology between BMI and NEU. As shown in Fig. 7, both genetic and residual effects on BMI are significantly modulated by individual NEU while there also is a strong genetic association between them. It was noted that in contrast to BMI-SMK analysis, the results between RNM and MRNM were similar, possibly reflecting different shared genetic and environmental architecture between BMI and NEU, compared to BMI and SMK. The estimated genetic architecture from BMI-NEU analyses is depicted in Fig. 7, Supplementary Data 6 and Supplementary Fig. 13. 

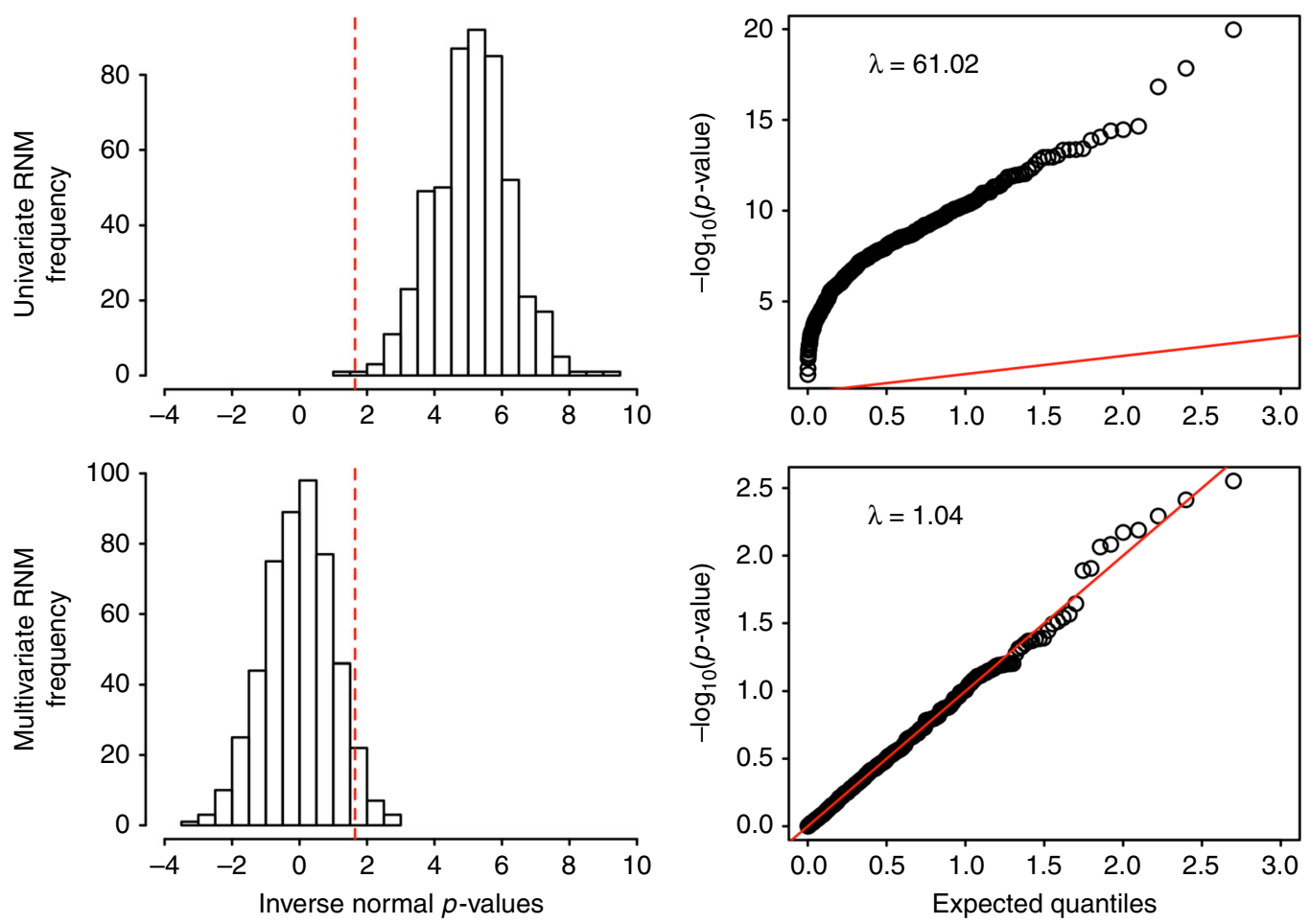

Fig. 4 Spurious signals generated by incorrect (univariate) model can be controlled by MRNM. Five hundred replicates of data were simulated under a null model that assumed genotype-covariate correlation but no genotype-covariate interaction. Simulation was based QCed ARIC data consisting 7,263 individuals and 583,058 SNPs. The model is specified as $\mathbf{y}=\boldsymbol{\alpha}_{\mathbf{0}}+\boldsymbol{\alpha}_{\mathbf{1}} \times \mathbf{c}+\mathbf{e}$ with $\mathbf{c}=\boldsymbol{\beta}+\boldsymbol{\varepsilon}$, all effects drawn from a multivariate normal distribution, where the variance-covariance structure between $\boldsymbol{\alpha}_{\mathbf{0}}, \boldsymbol{\beta}$, and $\boldsymbol{\alpha}_{\mathbf{1}}$ (in this order) is $\left[\begin{array}{ccc}1 & 0.5 & 0 \\ 0.5 & 1 & 0 \\ 0 & 0 & 0\end{array}\right]$ and that between $\mathbf{e}$ and $\boldsymbol{\varepsilon}$ is $\left[\begin{array}{cc}1 & 0.3 \\ 0.3 & 1\end{array}\right]$. For every replicate, a univariate RNM and a multivariate RNM were fitted separately to obtain a $p$-value for the $\mathrm{G}-\mathrm{C}$ interaction by comparing the null $\left(\mathrm{H}_{0}\right)$ and alternative hypothesis $\left(\mathrm{H}_{1}\right)$ model using a likelihood ratio test. For the univariate RNM, the $\mathrm{H}_{0}$ and $\mathrm{H}_{1}$ models were $\mathbf{y}=\boldsymbol{\alpha}_{\mathbf{o}}+\mathbf{e}$ and $\mathbf{y}=\boldsymbol{\alpha}_{\mathbf{o}}+\boldsymbol{\alpha}_{\mathbf{1}} \times \mathbf{c}+\mathbf{e}$. For the multivariate RNM, the $\mathrm{H}_{\mathrm{O}}$ and $\mathrm{H}_{1}$ models were $\mathbf{y}=\boldsymbol{\alpha}_{\mathbf{0}}+\mathbf{e}$ with $\mathbf{c}=\boldsymbol{\beta}+\boldsymbol{\varepsilon}$ and $\mathbf{y}=\boldsymbol{\alpha}_{\mathbf{0}}+\boldsymbol{\alpha}_{\mathbf{1}} \times \mathbf{c}+\mathbf{e}$ with $\mathbf{c}=\boldsymbol{\beta}+\boldsymbol{\varepsilon}$. The left panel shows the proportions of significant $p$-values, i.e., type I error rate, for both models, which are 0.998 (univariate RNM) and 0.046 (multivariate RNM). Note that $p$-values are inverse normal transformed, such that the statistical significance level, i.e., 1.65, shown as dashed lines, is equivalent to the 0.05 level before the transformation. The right panel is quantile-quantile (Q-Q) plots of $-\log 10$ ( $p$-values) from univariate RNM and multivariate RNM. $\lambda$ is the ratio of observed to expected median test statistic, which is inflated when using an inappropriate model

Lastly, we used PC1 as the covariate in the same analyses (Table 1) and as expected, found no significant interaction effects. Compared to SMK or NEU, the R-C interaction was dramatically less (Table 1 and Supplementary Data 6), probably because PC1 was calculated from genotype data for which the residual component was relatively small. We also found no evidence of G-C interaction, which was probably due to the fact that the sample was so homogeneous such that there was little power to detect interaction effects modulated by population difference.

The phenotypes of BMI showed some deviation from a normal distribution (kurtosis $=5.65$ and skewness $=1.08$ ). We explicitly tested the normality assumption using simulations, and found that rank-based inverse normal transformed (RINT) phenotypes could remedy type I error inflation due to non-normality (Supplementary Note 2). However, the conclusions drawn from our analyses were the same when either raw or RINT phenotypes are used (Table 1 vs. and Supplementary Table 8). Therefore, we report significances and estimations based on raw phenotypes here.

Inflated residual variance using GREML with real data. We observed in simulation data that residual variances for a trait estimated from LDSC or GREML were inflated when there were G-C or R-C interactions (Fig. 5 and Supplementary Table 7), and this led to underestimates of SNP heritability. Hence, with real data, we tested the differences in the estimates of residual variances for BMI estimated from GREML and RNM (Table 2). For this real data analysis, we could not assess LDSC performance because it did not provide the standard error of estimated residual variance that was required for testing a statistical difference (Supplementary Note 3). For SMK and NEU that had significant interaction effects, the estimated residual variances from GREML were significantly higher than those from RNM (1.89\% difference with $p$-value $=5.99 \mathrm{E}$ - 04 and $2.04 \%$ difference with $p$-value $=$ 7.12E-03, using a Wald test) (Table 2). As expected, there was no significant difference between the models when PC1 was considered as the covariate, because it had no interaction effects. We also fitted both SMK and NEU simultaneously and found that the difference between estimated residual variances from GREML and RNM was increased (3.28\% with p-value $1.57 \mathrm{E}-04$ using a Wald test) (Table 2). The estimated variance components for the interaction effects from the joint model (Supplementary Table 9) and the separate models (M4 in Supplementary Data 6) did not differ. We also observed that the estimated genetic variance varied little between using GREML or RNM (Fig. 5 and M3 in Supplementary Data 1 and 2), hence biased residual variance directly caused biased SNP-based heritability estimates. Inflated residual variance therefore underestimated SNP-based heritability, as also observed from an extensive meta-analysis across diverse 

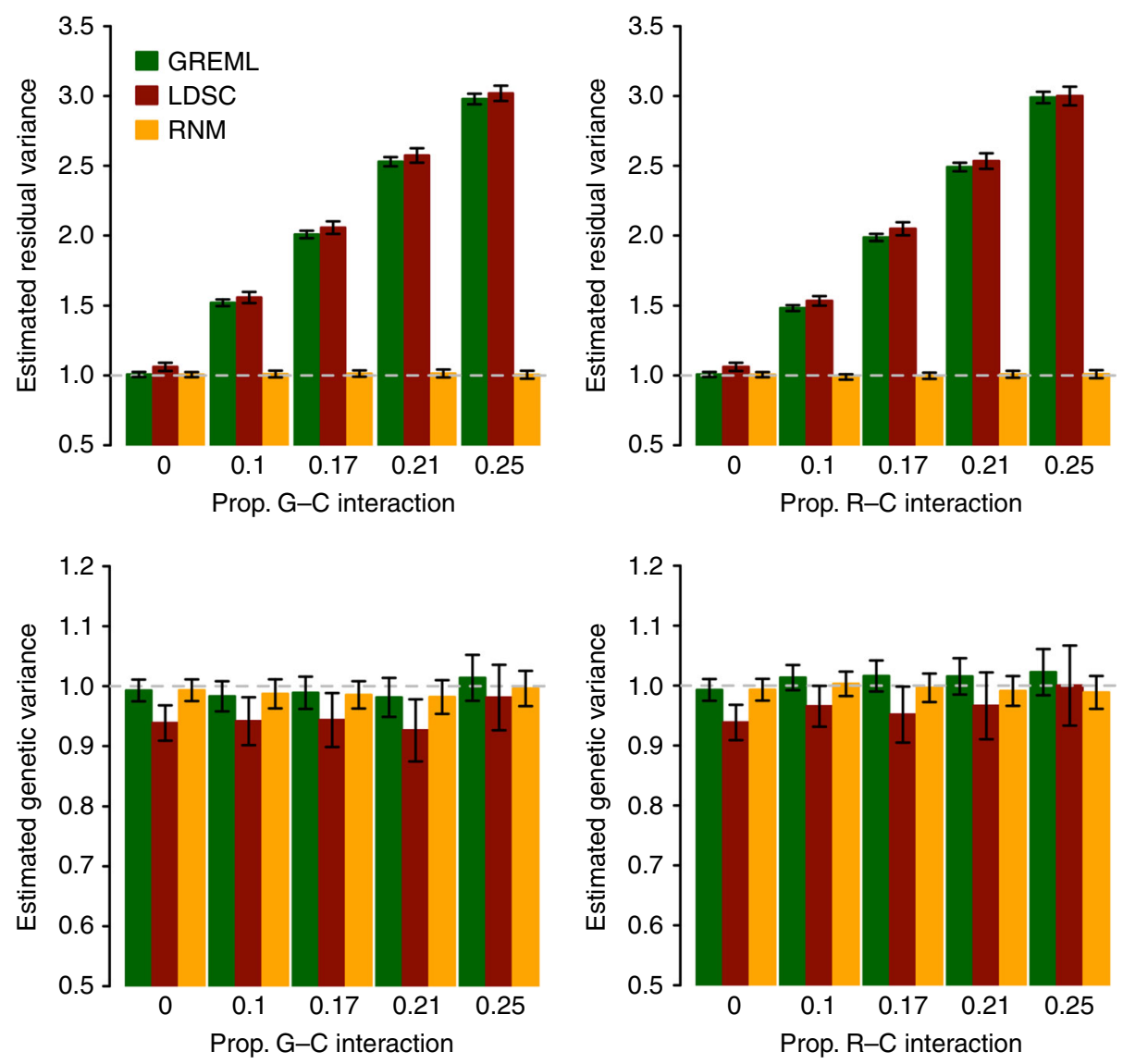

Fig. 5 Estimated residual and genetic variances from data simulated under interaction models. Prop. G-C or R-C interaction is the proportion of variance due to $\boldsymbol{\alpha}_{\mathbf{1}}$ or $\boldsymbol{\tau}_{\mathbf{1}}$ (see below) in the total phenotypic variance (i.e., $\operatorname{var}\left(\boldsymbol{\alpha}_{\mathbf{1}}\right) / \operatorname{var}(\mathbf{y})$ in G-C interaction model or $\operatorname{var}\left(\boldsymbol{\tau}_{\mathbf{1}}\right) / \operatorname{var}(\mathbf{y})$ in $\mathrm{R}-\mathrm{C}$ interaction model). Simulation for G-C interaction ( $\boldsymbol{\alpha}_{\mathbf{1}}$ ) (left panels): The phenotype data were generated using $\mathbf{y}=\boldsymbol{\alpha}_{\mathbf{0}}+\boldsymbol{\alpha}_{\mathbf{1}} \times \mathbf{c}+\mathbf{e}$ with $\mathbf{c}=\boldsymbol{\beta}+\boldsymbol{\varepsilon}$, all effects drawn from a multivariate normal distribution. The variance-covariance structure of $\boldsymbol{\alpha}_{\mathbf{0}}, \boldsymbol{\beta}$, and $\boldsymbol{\alpha}_{\mathbf{1}}$ (in this order) is $\left[\begin{array}{ccc}1 & 0 & 0.05 \\ 0 & 1 & 0 \\ 0.05 & 0 & \operatorname{var}\left(\boldsymbol{\alpha}_{1}\right)\end{array}\right]$ with var( $\left.\boldsymbol{\alpha}_{\mathbf{1}}\right)=0,0.25,0.5$, 0.75 and 1 and that for $\mathbf{e}$ and $\boldsymbol{\varepsilon}$ is $\left[\begin{array}{ll}1 & 0 \\ 0 & 1\end{array}\right]$. Simulation for R-C interaction $\left(\boldsymbol{\tau}_{\mathbf{1}}\right)$ (right panels): The phenotype data were generated using $\mathbf{y}=\boldsymbol{\alpha}_{\mathbf{0}}+\boldsymbol{\tau}_{\mathbf{0}}$ $+\boldsymbol{\tau}_{\mathbf{1}} \times \mathbf{c}$ with $\mathbf{c}=\boldsymbol{\beta}+\boldsymbol{\varepsilon}$, all effects drawn from a multivariate normal distribution. The variance-covariance structure of $\boldsymbol{\alpha}_{\mathbf{0}}$ and $\boldsymbol{\beta}$ is $\left[\begin{array}{ll}1 & 0 \\ 0 & 1\end{array}\right]$ and that of $\boldsymbol{\tau}_{\mathbf{0}}, \boldsymbol{\varepsilon}$, and $\boldsymbol{\tau}_{\mathbf{1}}$ (in this order) is $\left[\begin{array}{ccc}1 & 0 & 0.05 \\ 0 & 1 & 0 \\ 0.05 & 0 & \operatorname{var}\left(\boldsymbol{\tau}_{1}\right)\end{array}\right]$ with $\operatorname{var}\left(\boldsymbol{\tau}_{\mathbf{1}}\right)=0,0.25,0.5,0.75$ and 1 . The error bar is a 95\% confidence interval, which was estimated over 100 replicates. The model for GREML is $\mathbf{y}=\boldsymbol{\alpha}_{\mathbf{0}}+\mathbf{e}$ and the model for RNM in the left panel is $\mathbf{y}=\boldsymbol{\alpha}_{\mathbf{0}}+\boldsymbol{\alpha}_{\mathbf{1}} \times \mathbf{c}+\mathbf{e}$. The model for RNM in the right panel is $\mathbf{y}=\boldsymbol{\alpha}_{\mathbf{0}}+\boldsymbol{\tau}_{\mathbf{0}}+\boldsymbol{\tau}_{\mathbf{1}} \times \mathbf{c}$. Simulation was based QCed ARIC data consisting 7,263 individuals and 583,058 SNPs

study-cohorts $23,29,30$ that possibly increased the heterogeneity of covariates shared by the study-samples, hence increased the variance due to $\mathrm{G}-\mathrm{C}$ and/or $\mathrm{R}-\mathrm{C}$ interactions (Fig. 8). When comparing MRNM and MVGREML, the results did not differ much (Supplementary Table 10) although there were additional parameters such as $\operatorname{cov}\left(\boldsymbol{\alpha}_{1}, \boldsymbol{\beta}\right)$ and $\operatorname{cov}\left(\boldsymbol{\tau}_{1}, \boldsymbol{\varepsilon}\right)$ that were not explicitly parameterised in GREML. We did not fit multiple covariates jointly in MRNM because of our focus on SNP-based heritability comparisons based on univariate models (i.e., GREML vs. RNM) and due to the need to control computational demands.

Meta-analysis approach and validation using UK Biobank data. For very large data sets, our proposed approach may become computationally infeasible (see Supplementary Table 11 for computational requirements). A solution could be to divide the data in various subsets and undertake a meta-analysis. We show that a meta-analysis ${ }^{31}$ of GCCI and RCCI results across different data subsets is useful and reliable (Supplementary Note 4).

\section{Discussion}

Complex traits are determined by both genetic and environmental effects. Some environmental covariates of complex traits may themselves be determined by genetic and non-genetic factors. Genotype-covariate correlation and interactions (GCCI) and residual-covariate correlation and interaction effects (RCCI) may be important underlying factors shaping complex trait phenotypes $^{32}$, yet not many studies have conducted analyses to detect these effects jointly in one model because of a lack of proper analysis models. In this study, we propose a flexible (multivariate) RNM to estimate genotype-covariate correlation and interactions and residual-covariate correlation and interaction effects for complex traits, which is powerful and reliable. The key findings are summarised as follows:

1. For continuous covariates, the proposed MRNM is a more appropriate model, compared to RR-GREML and GCIGREML. 
Table 1 P-values of likelihood ratio tests for model comparisons in UK Biobank analyses of BMI

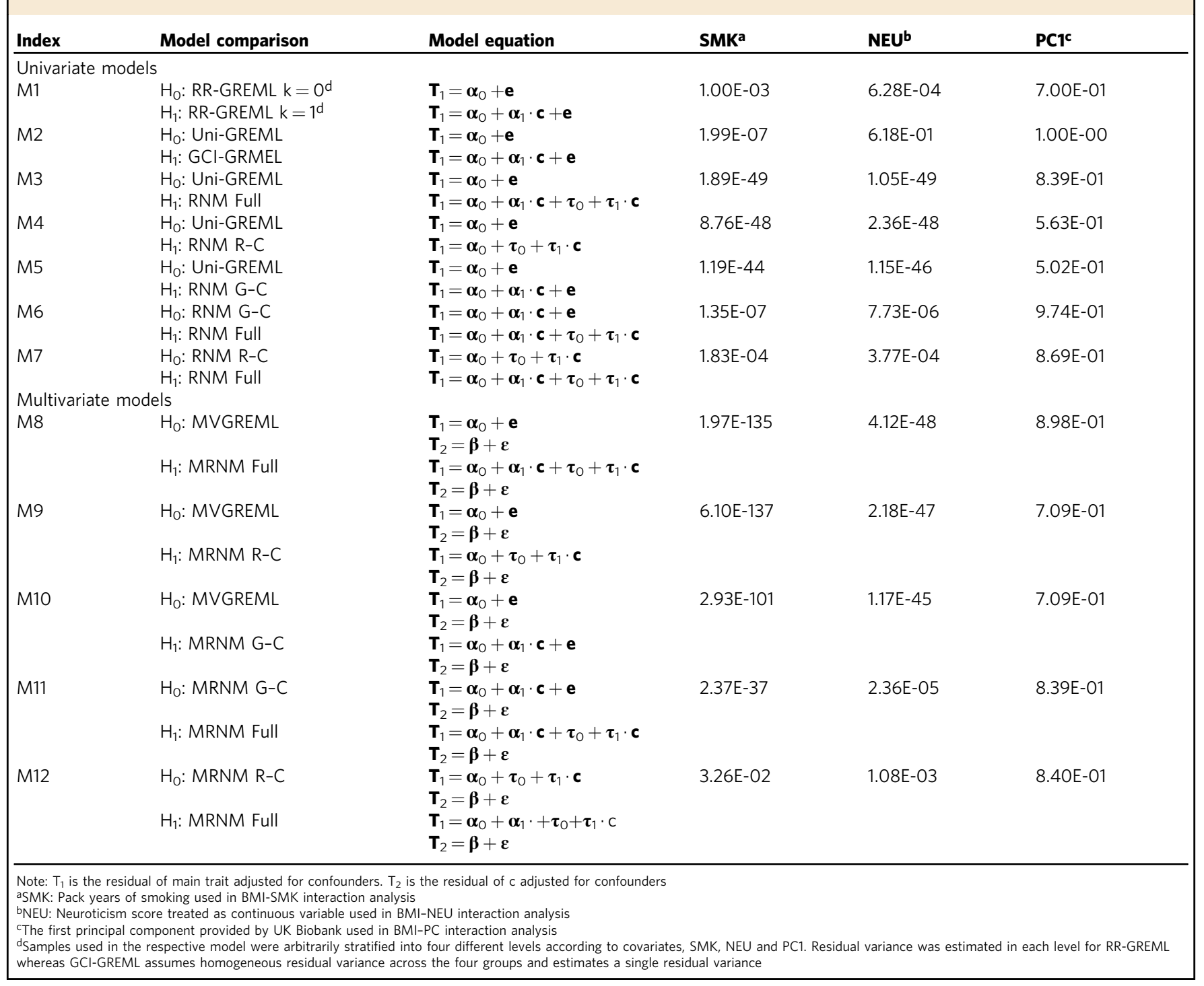

2. Covariates can be regulated by genetic and environmental factors that are possibly shared with the main response (GCCI and RCCI effects), which is the most plausible mechanism for many complex traits. It is desirable to model GCCI and RCCI effects appropriately (using multivariate RNM).

3. SNP-based heritability estimates from standard additive models should be carefully interpreted or revisited if covariate information is available.

4. The proposed models can be applied to large-scale biobank data by meta-analysis of results from sub-samples, for which the analyses are computationally feasible.

The existing methods for estimation of $\mathrm{G}-\mathrm{C}$ interactions, i.e., RR-GREML and GCI-GREML, require that the outcome of interest (e.g., BMI) should be stratified into multiple discrete groups. The discrete grouping ignores the difference of covariate values for the individuals within each group, and results in some loss of information. In contrast, RNM and MRNM use a flexible model to fit a continuous covariate. Based on the analysis method that we believe to be the most appropriate for the data (MRNM) the $\mathrm{G}-\mathrm{C}$ interaction estimate was much reduced and only borderline significant while $\mathrm{R}-\mathrm{C}$ interaction was much more significant.
In the presence of $\mathrm{G}-\mathrm{C}$ or $\mathrm{R}-\mathrm{C}$ interactions, estimated SNPbased heritability of the main response variable by GREML or LDSC could be biased. The biased estimates reflect that the interaction effects are absorbed by residual variance and the overall estimated residual variance was inflated. The residual variance estimated from GREML was significantly higher than that from RNM for the BMI-SMK, BMI-NEU or BMI-SMK/NEU analysis using the real data. Currently reported SNP-based heritabilites estimated based on meta analysis of GWAS summary statistics from diverse study-cohorts tend to be lower when the number of study-cohorts is larger (as a proxy of heterogeneity) (Fig. 8), which can be partly explained by not properly modelling $\mathrm{G}-\mathrm{C}$ and $\mathrm{R}-\mathrm{C}$ interactions. This observation has an important implication because estimates from such meta-analyses (using LDSC) should be carefully interpreted when known key covariates were not included in the GWAS analysis model that generated the input for the LDSC analysis.

In this study, we found a strong negative $\mathrm{R}-\mathrm{C}$ correlation and weak positive $\mathrm{G}-\mathrm{C}$ correlation between $\mathrm{BMI}$ and smoking, which may support the phenomenon observed in several studies that heavier smokers tend to have lower BMI. The $\mathrm{R}-\mathrm{C}$ interaction was shown to be highly significant. This suggests that the information about $\mathrm{R}-\mathrm{C}$ interaction component is crucial such that that 


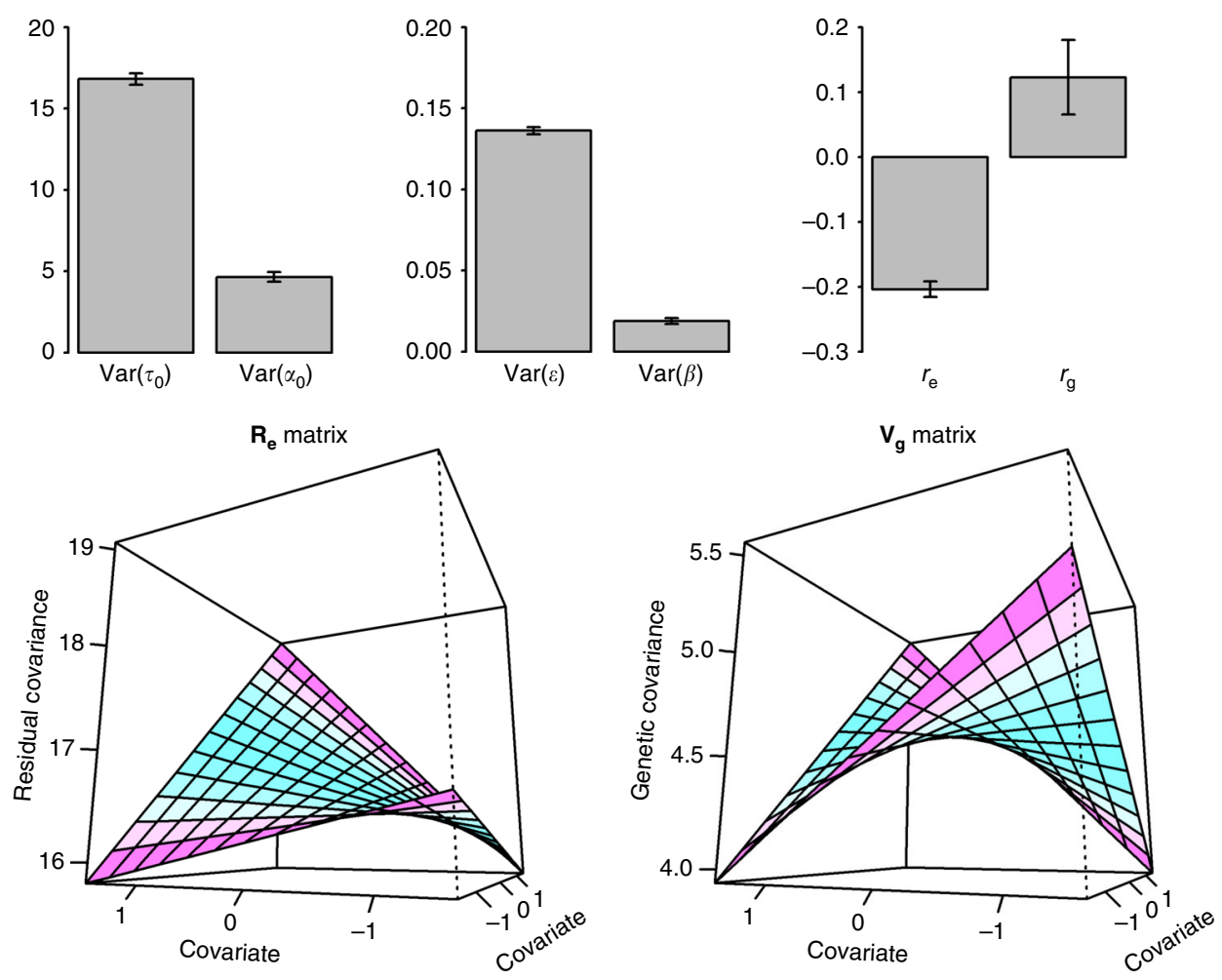

Fig. 6 Estimated variance components and correlations from MRNM for BMI-SMK analysis. $\operatorname{Var}\left(\boldsymbol{\tau}_{0}\right)$ : Estimated residual variance for BMI as the main outcome. $\operatorname{Var}\left(\boldsymbol{\alpha}_{0}\right)$ : Estimated genetic variance for BMI as the main outcome. $\operatorname{Var}(\boldsymbol{\varepsilon})$ : Estimated residual variance for SMK as the covariate. $\operatorname{Var}(\boldsymbol{\beta})$ : Estimated genetic variance for SMK as the covariate. $r_{\mathrm{e}}$ : Estimated residual correlation between BMI and SMK. $r_{\mathrm{g}}$ : Estimated genetic correlation between BMI and SMK. Error bars are $95 \%$ confidence interval. $\mathbf{R}_{\mathbf{e}}$ matrix is the residual (co)variance structure between different covariate levels (see Eq. 4), which is derived using estimated random regression coefficients and polynomial matrix as $\mathbf{R}_{\mathbf{e}}=\mathbf{\Phi} \mathbf{M}_{\mathbf{y}} \boldsymbol{\Phi}^{\prime}$. $\boldsymbol{\Phi}$ is the matrix of polynomials evaluated at given covariate values, where entries of the first column are all $\mathbf{1 s}$ and the second column is the standardised covariates of respective individuals. $\mathbf{M}_{\mathbf{y}}$ is the variance-covariance matrix of estimated random regression coefficients from MRNM as $\mathbf{M}_{\mathbf{y}}=\left[\begin{array}{cc}\operatorname{var}\left(\boldsymbol{\tau}_{0}\right) & \operatorname{cov}\left(\boldsymbol{\tau}_{0}, \boldsymbol{\tau}_{1}\right) \\ \operatorname{cov}\left(\boldsymbol{\tau}_{0}, \boldsymbol{\tau}_{1}\right) & \operatorname{var}\left(\boldsymbol{\tau}_{1}\right)\end{array}\right]=\left[\begin{array}{cc}16.81(\mathrm{SEO} 0.18) & 0.41(\mathrm{SEO} 0.12) \\ 0.41(\mathrm{SEO} .12) & 0.42(\mathrm{SEO} 0.16)\end{array}\right] . \mathbf{V}_{\mathbf{g}}$ matrix in is the genetic (co)variance structure between different covariate levels (see Eq. 2), which is derived based on the estimated random regression coefficients and polynomial matrix as $\mathbf{V}_{\mathbf{g}}=\mathbf{\Phi} \mathbf{K}_{\mathbf{y}} \boldsymbol{\Phi}^{\prime}$. $\boldsymbol{\Phi}$ is the matrix of polynomials evaluated at given covariate values, where entries of the first column are all $\mathbf{1 s}$ and the second column is the standardised covariates of respective individuals. $\mathbf{K}_{\mathbf{y}}$ is the variance-covariance matrix of random regression coefficients estimated from MRNM as $\mathbf{K}_{\mathbf{y}}=\left[\begin{array}{cc}\operatorname{var}\left(\boldsymbol{\alpha}_{0}\right) & \operatorname{cov}\left(\boldsymbol{\alpha}_{0}, \boldsymbol{\alpha}_{1}\right) \\ \operatorname{cov}\left(\boldsymbol{\alpha}_{0}, \boldsymbol{\alpha}_{1}\right) & \operatorname{var}\left(\boldsymbol{\alpha}_{1}\right)\end{array}\right]=\left[\begin{array}{cc}4.66(\text { SEO.15) } & 0.07(\text { SEO.10) } \\ 0.07(\text { SEO.10) } & 0.30(\text { SEO.12) }\end{array}\right]$

the main phenotypes (BMI) can be possibly controlled by changing the covariate (SMK), provided that the covariate is modifiable. In this example, the implication is that the intervention of increasing smoking could be used to control $\mathrm{BMI}^{33}$. While in this example the advice may not be practical for other health reasons, the principle can be used to other traits and diseases with modifiable covariates. The information from the G-C correlation and interaction can be useful for an early intervention (e.g., genomic medicine) although the magnitude of the effects is relatively small, compared to $\mathrm{R}-\mathrm{C}$ components. We also investigated NEU and found strong $\mathrm{G}-\mathrm{C}$ and $\mathrm{R}-\mathrm{C}$ interactions, indicating the personality trait NEU is a major covariate influencing the environmental factor for BMI as well as revealing a novel genetic architecture of BMI to interact with different levels of NEU (Fig. 7). Both genetic and residual variances of BMI are significantly modulated by NEU, as well as there is significant genetic correlation between BMI and NEU. We included analyses using PC1 as the covariate in the model. Because of little variance among the homogenous sample, there were no significant interactions (Table 1 and Supplementary Table 8). In other circumstances, for example when using diverse samples from the population or even across different ethnic populations, then analyses that fit $\mathrm{PC1}$ as a covariate might generate significant interaction estimates.

Our analytical framework could be extended to consider genotype-by-genotype interactions, i.e., epistasis, such that an interaction between the genetic component of a trait and a covariate might be partitioned as interaction variances in the proposed model. However, for the sorts of phenotypes considered here there is no power to disentangle epistasis from other interactions with the data available to us. However, the proposed approach may have important utility in the context of geneexpression, transcriptome and methylation data as novel covariates, for which the specific genetic architecture may be powered to detect epistatic interactions and correlations, and may merit future study. It is possible to use models that fit multiple covariates simultaneously as we did for fitting both SMK and NEU jointly (Table 2), which increase the proportion of the total phenotypic variance explained by the interaction components. Genomic partitioning analyses to describe GCCI and RCCI effects across the genome will be also useful to shed light on the latent genetic architecture of complex traits and diseases, which is possible by using the proposed approaches in this study. 

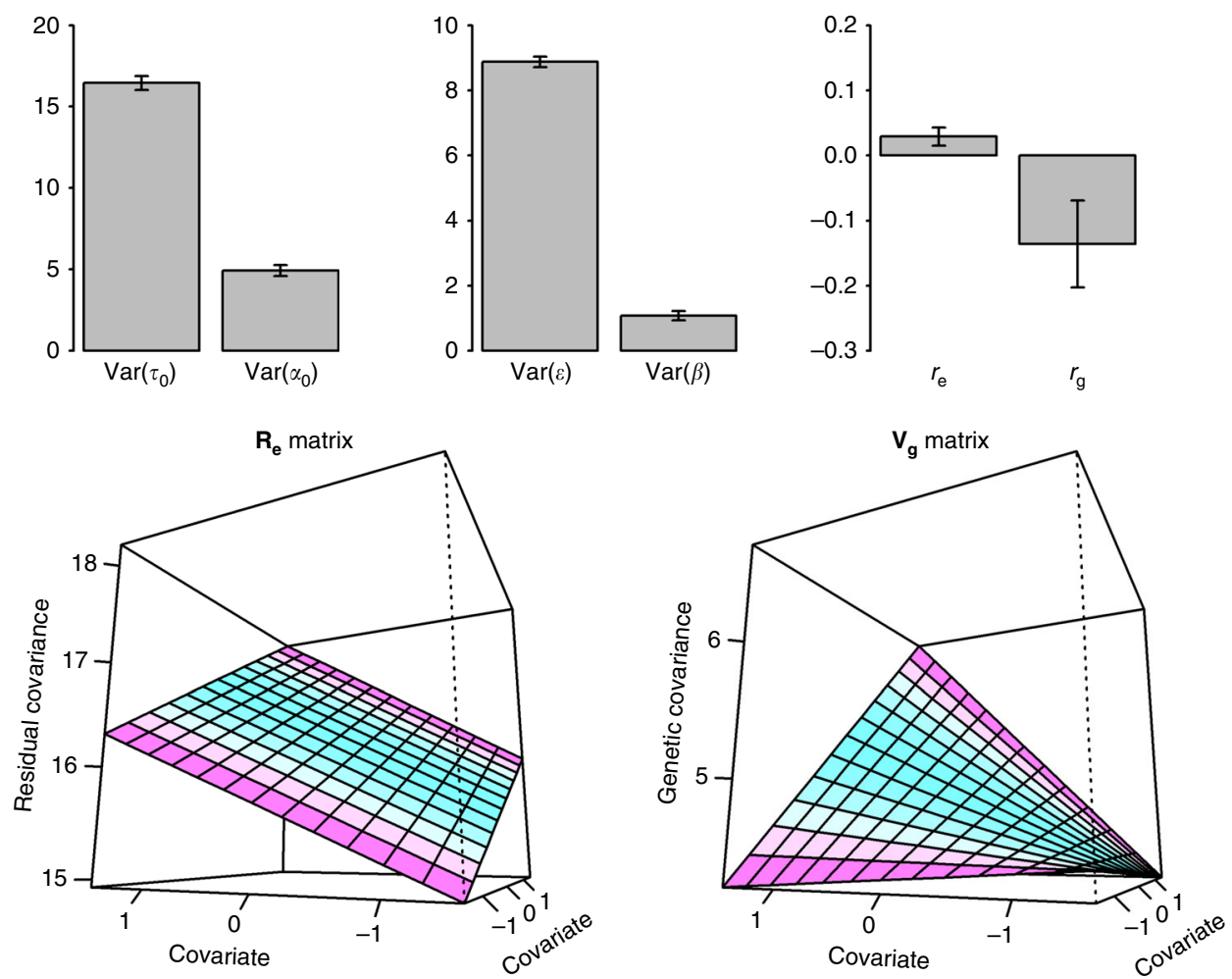

Fig. 7 Estimated variance components and correlations from MRNM for BMI-NEU analysis. $\operatorname{Var}\left(\boldsymbol{\tau}_{0}\right)$ : Estimated residual variance for BMI as the main outcome. $\operatorname{Var}\left(\boldsymbol{\alpha}_{0}\right)$ : Estimated genetic variance for BMI as the main outcome. $\operatorname{Var}(\boldsymbol{\varepsilon})$ : Estimated residual variance for NEU as the covariate. $\operatorname{Var}(\boldsymbol{\beta})$ : Estimated genetic variance for NEU as the covariate. $r_{\mathrm{e}}$ : Estimated residual correlation between BMI and NEU. $r_{\mathrm{g}}$ : Estimated genetic correlation between BMI and NEU. Error bars are $95 \%$ confidence interval. $\mathbf{R}_{\mathbf{e}}$ matrix is the residual (co)variance structure between different covariate levels (see Eq. 4), which is derived based on the estimated random regression coefficients and polynomial matrix as $\mathbf{R}_{\mathbf{e}}=\mathbf{\Phi} \mathbf{M}_{\mathbf{y}} \boldsymbol{\Phi}^{\prime}$. $\boldsymbol{\Phi}$ is the matrix of polynomials evaluated at given covariate values, where entries of the first column are all $\mathbf{1 s}$ and the second column is the standardised covariates of respective individuals. $\mathbf{M}_{\mathbf{y}}$ is the variance-covariance matrix of random regression coefficients estimated from MRNM as $\mathbf{M}_{\mathbf{y}}=\left[\begin{array}{cc}\operatorname{var}\left(\boldsymbol{\tau}_{0}\right) & \operatorname{cov}\left(\boldsymbol{\tau}_{0}, \boldsymbol{\tau}_{1}\right) \\ \operatorname{cov}\left(\boldsymbol{\tau}_{0}, \boldsymbol{\tau}_{1}\right) & \operatorname{var}\left(\boldsymbol{\tau}_{1}\right)\end{array}\right]=\left[\begin{array}{cc}16.45(\mathrm{SEO} 0.21) & 0.54(\mathrm{SEO} 0.11) \\ 0.54(\operatorname{SEO} 0.11) & 0.06(\mathrm{SEO} 0.18)\end{array}\right] . \mathbf{v}_{\mathbf{g}}$ matrix in is the genetic (co)variance structure between different covariate levels (see Eq. 2), which is derived based on the estimated random regression coefficients and polynomial matrix as $\mathbf{V}_{\mathbf{g}}=\mathbf{\Phi} \mathbf{K}_{\mathbf{y}} \boldsymbol{\Phi}^{\prime}$. $\boldsymbol{\Phi}$ is the matrix of polynomials evaluated at given covariate values, where entries of the first column are all $\mathbf{1}$ and the second column is the standardised covariates of respective individuals. $\mathbf{K}_{\mathbf{y}}$ is the variance-covariance matrix of random regression coefficients estimated from MRNM as $\mathbf{K}_{\mathbf{y}}=\left[\begin{array}{cc}\operatorname{var}\left(\boldsymbol{\alpha}_{0}\right) & \operatorname{cov}\left(\boldsymbol{\alpha}_{0}, \boldsymbol{\alpha}_{1}\right) \\ \operatorname{cov}\left(\boldsymbol{\alpha}_{0}, \boldsymbol{\alpha}_{1}\right) & \operatorname{var}\left(\boldsymbol{\alpha}_{1}\right)\end{array}\right]=\left[\begin{array}{cc}4.94(\text { SEO.17) } & 0.38(\text { SEO.11) } \\ 0.38(\text { SEO.11) } & 0.28(\text { SEO.13) }\end{array}\right]$

Table 2 The difference between residual variances of BMI estimated from RNMa and GREMLb

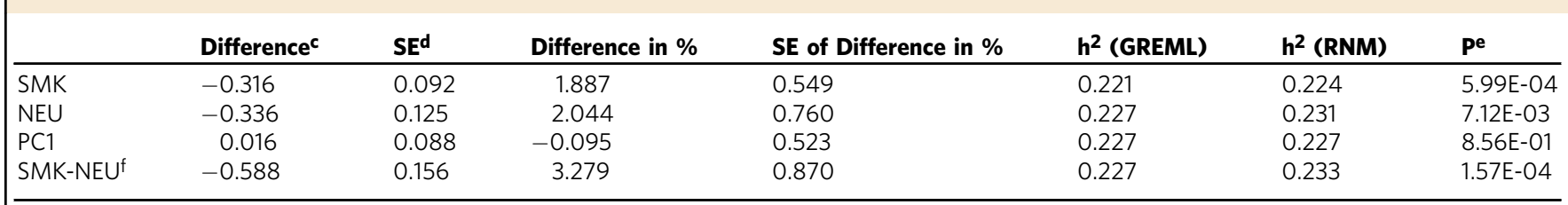

alternative model $(\mathrm{H} 1)$ of $\mathrm{M} 3$ in Table 1

b Null model $(\mathrm{HO})$ of $\mathrm{M} 3$ in Table 1

'Difference $=$ the residual variance estimated from RNM - the residual variance estimated from univariate GREML

dStandard error of the difference was calculated based on the theory in Supplementary Note 3

eP-value was obtained based a two-tailed Wald test using the difference of residual variances and its SE

fThe model jointly fitted both SMK and NEU as multiple covariates (see Methods)

An alternative approach to disentangle interaction from association is through the classical structural equation models ${ }^{11}$ applied to twin- or pedigree-based data. However, availability of such data is limited, restricting our ability to study GCCI effects for a wide range of complex traits and covariates. For example, phenotypes moderated by ancestry components (e.g., ethnic composition in humans or breed composition in animals) cannot be studied by an approach that is based on twins or relatives. It is also difficult to disentangle the genetic and shared environmental effects when using a pedigree-based approach. Standard REML packages (e.g., ASReml ${ }^{34}$ ) can be used to test the GCCI effects although it is questionable that the classical REML algorithm, which has been optimised for pedigree-based studies, can be computationally tractable when fitting genetic covariance structures based on genomic information ${ }^{17}$. Therefore, it may be infeasible investigate the GCCI effects using the classical REML 


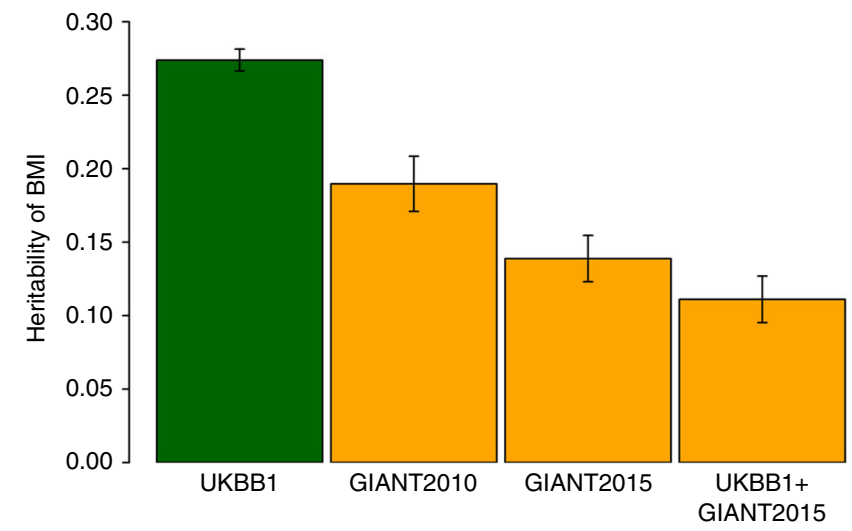

Fig. 8 Estimated SNP-based heritability of BMI decreases with increasing numbers of cohorts. The UKBB1 estimate was reported by Ge et al. ${ }^{23}$, which used GWAS summary statistics based on the samples from the first release of UK Biobank. The GIANT2010 and GIANT 2015 estimates were reported by Duncan et al. ${ }^{29}$, which used GWAS summary statistics based on the GIANT consortium samples from $\sim 80$ to 125 cohorts, respectively. The UKBB1 + GIANT2015 estimate was reported by Ni et al. ${ }^{30}$. Bars are $95 \%$ confidence interval

packages although they have been applied widely in livestock 35,36 and ecological genetics ${ }^{13,37,38}$ to explore the phenotype-genotype relationship across environmental gradients. When extending analyses to cover large-scale data such as the UK Biobank ${ }^{24}$, it is essential to develop computationally efficient methods that also correctly capture the GCCI effects, based on genomic information.

There are a number of limitations and caveats in this study. First, we only considered interaction of order $k=1$ for both G-C and $\mathrm{R}-\mathrm{C}$ interactions. Further study is required to validate performance with higher order interactions to generalise the proposed approach. Second, our approaches are flexible, but computationally demanding. For a large data set or when conducting randomisation tests, e.g., permutation or bootstrapping, it may not be computationally feasible to conduct an analysis within a reasonable time. Subsampling and meta-analysis approaches can reduce analysis time, however, the power is notably reduced, compared to when using the whole data (Supplementary Figs. 14 and 15). Third, the proposed methods do not estimate the direction of causality that can be determined by existing methods, e.g., Mendelian randomisation. Fourth, in application to real data we do not take account of ascertainment biases that may generate interactions and correlations in the sample of data which means that our results may not be representative of the populations from which the samples are drawn ${ }^{39,40}$. Lastly, when there are assumption violations, such as non-normality, correlation between random effects or wrong estimation models due to missing or misspecified information, one needs to carefully check model performance (as in the Supplementary Notes 2 and 5). For non-normally distributed traits, we recommend using RINT phenotypes to check the robustness of estimated interaction effects. Interaction effects will require careful interpretation, particularly for $\mathrm{R}-\mathrm{C}$ effects which can be caused by residual heteroscedasticity by covariates ${ }^{41}$.

In conclusion, we showed that the multivariate RNM is able to effectively disentangle interaction from correlation and to generate unbiased estimates for $\mathrm{G}-\mathrm{C}$ and $\mathrm{R}-\mathrm{C}$ components. The concept of GCCI and RCCI is more plausible in explaining the genetic architecture of complex traits associated/interacted with covariates, which will shift the paradigm from a univariate to multivariate framework and from linear to non-linear models in complex trait analyses.

\section{Methods}

UK Biobank's scientific protocol and operational procedures were reviewed and approved by the North West Multi-centre Research Ethics Committee (MREC), National Information Governance Board for Health \& Social Care (NIGB), and Community Health Index Advisory Group (CHIAG). The protocol of Atherosclerosis Risk in Communities Study (ARIC) study has been reviewed and approved by the Institutional Review Boards (IRB) of each participating institution, including the IRBs of the University of Minnesota, Johns Hopkins University, University of North Carolina, University of Mississippi Medical Center, and Wake Forest University. Research Ethics approval was obtained from University of South Australia Human Research Ethics Committee (HREC).

Reaction norm model (RNM). We only consider interaction of order $k=1$ in the following equations. More general equations with a higher order can be found in Supplementary Note 1 .

To account for phenotypic plasticity and norms of reaction in response to different covariate or environmental conditions among samples ${ }^{35,36}$, the dependent variable for individual $i$ can be modelled as

$$
y_{i}=b_{i}+g_{i}+e_{i}=b_{i}+\alpha_{i 0}+\alpha_{i 1} \cdot c_{i}+e_{i}
$$

where $y_{i}$ is the phenotypic observation, $b_{i}$ represents fixed effects, $g_{i}$ is the random genetic effect, $\alpha_{i 0}$ and $\alpha_{i 1}$ are the zero and first order of random regression coefficients, $c_{\mathrm{i}}$ is the covariate value, and $e_{i}$ is the residual effect for the $i$ th individual. Assuming that each individual has unique covariate value, the variance-covariance matrix of observed phenotypes $\left(y_{\mathrm{i}}\right)$ is

$$
\operatorname{var}(\mathbf{y})=\left[\begin{array}{ccc}
\mathbf{Z}_{1} \mathbf{A} \sigma_{g_{1}}^{2} \mathbf{Z}_{1}^{\prime}+\mathbf{Z}_{1} \mathbf{I} \sigma_{e_{1}}^{2} \mathbf{Z}_{1}^{\prime} & \cdots & \mathbf{Z}_{1} \mathbf{A} \sigma_{g_{1, N}} \mathbf{Z}_{N}^{\prime}+\mathbf{Z}_{1} \mathbf{I} \sigma_{e_{1, N}} \mathbf{Z}_{N}^{\prime} \\
\vdots & \ddots & \vdots \\
\mathbf{Z}_{\mathrm{N}} \mathbf{A} \sigma_{g_{1, N}} \mathbf{Z}_{1}^{\prime}+\mathbf{Z}_{\mathrm{N}} \mathbf{I} \sigma_{e_{1, N}} \mathbf{Z}_{1}^{\prime} & \cdots & \mathbf{Z}_{N} \mathbf{A} \sigma_{g_{N}}^{2} \mathbf{Z}_{\mathrm{N}}^{\prime}+\mathbf{Z}_{N} \mathbf{I} \sigma_{e_{N}}^{2} \mathbf{Z}_{N}^{\prime}
\end{array}\right],
$$

where $\mathbf{A}$ is the $N \times N$ genomic relationship matrix based on genome-wide SNP information, $\mathbf{Z}_{\mathrm{i}}$ is an incidence matrix for $g_{i}$, and $\mathbf{I}$ is an $N \times N$ identity matrix. The terms $\sigma_{g_{i}}^{2}$ and $\sigma_{e_{i}}^{2}$ denote the genetic and residual variances at the covariate level for individual $i$. The terms $\sigma_{g_{i j}}$ and $\sigma_{e_{i j}}$ indicate the genetic and residual covariance between the covariate levels for individual $i$ and $j(i=1, \ldots, N$, and $j=1, \ldots, N)$, respectively ${ }^{17}$. The random genetic and residual effect are assumed following a normal distribution with mean as zero and variance as $\mathbf{A} \sigma_{g}^{2}$ and $\mathbf{I} \sigma_{e}^{2}$. The random genetic effect, $g_{i}$, can be regressed on the covariate gradient (reaction norm), which can be efficiently modelled with random regression coefficients. The variance-covariance matrix of random regression coefficients $(\mathbf{K})$ is

$$
\mathbf{K}=\operatorname{cov}\left(\boldsymbol{\alpha}_{0}, \boldsymbol{\alpha}_{1}\right)=\left[\begin{array}{cc}
\operatorname{var}\left(\boldsymbol{\alpha}_{0}\right) & \operatorname{cov}\left(\boldsymbol{\alpha}_{0}, \boldsymbol{\alpha}_{1}\right) \\
\operatorname{cov}\left(\boldsymbol{\alpha}_{0}, \boldsymbol{\alpha}_{1}\right) & \operatorname{var}\left(\boldsymbol{\alpha}_{1}\right)
\end{array}\right]
$$

where $\boldsymbol{\alpha}_{0}$ and $\boldsymbol{\alpha}_{1}$ are the zero and first order random regression coefficients. The genetic (co)variance matrix of genetic effects between $N$ individuals or $N$ covariate values (because each individual has unique covariate value) is a function of random regression coefficients and polynomials, which can be expressed as

$$
\mathbf{v}_{\mathbf{g}}=\boldsymbol{\Phi} \mathbf{K} \boldsymbol{\Phi}^{\prime}=\left[\begin{array}{ccc}
\sigma_{g_{1}}^{2} & \cdots & \sigma_{g_{1, N}} \\
\vdots & \ddots & \vdots \\
\sigma_{g_{N, 1}} & \cdots & \sigma_{g_{N}}^{2}
\end{array}\right]
$$

where $\Phi$ is the $N \times 2$ matrix of the zero and first order polynomials of $N$ covariate values, that is $\Phi_{i}=\left[c_{i}^{0}, c_{i}^{1}\right]$.

Given that this model does not explicitly parameterise the correlation between $y_{i}$ and $c_{i}$, it naively assumes that $y_{i}$ and $c_{i}$ are uncorrelated. For this reason, this model is also referred to as a genotype-covariate interaction ( $\mathrm{G}-\mathrm{C}$ interaction) model.

Multivariate reaction norm model (MRNM). The naïve assumption of the univariate RNM (or G-C interaction model) that $y_{i}$ and $c_{\mathrm{i}}$ are uncorrelated is often violated. In a more proper model, the covariate value for individual $i$ is decomposed as $c_{i}=\mu_{i}+\beta_{i}+\varepsilon_{i}$, where $\mu_{i}$ is fixed effects, $\beta_{i}$ is the random genetic effect, and $\varepsilon_{\mathrm{i}}$ is the residual effect. When considering the main response $(\mathbf{y})$ and covariate (c) jointly in a multivariate model, the variance-covariance matrix of observed phenotypes $y_{i}$ and $c_{i}$ is

$\operatorname{cov}(\mathbf{y}, \mathbf{c})=\left[\begin{array}{cccc}\mathbf{Z}_{1} \mathbf{A} \sigma_{g_{1}}^{2} \mathbf{Z}_{1}^{\prime}+\mathbf{Z}_{1} \mathbf{I} \sigma_{e_{1}}^{2} \mathbf{Z}_{1}^{\prime} & \cdots & \mathbf{Z}_{1} \mathbf{A} \sigma_{g_{1, N}} \mathbf{Z}_{N}^{\prime}+\mathbf{Z}_{1} \mathbf{I} \sigma_{e_{1, N}} \mathbf{Z}_{N}^{\prime} & \mathbf{Z}_{1} \mathbf{A} \sigma_{g_{1}, \beta} \mathbf{Z}_{c}^{\prime}+\mathbf{Z}_{1} \mathbf{I} \sigma_{e_{1}, \varepsilon} \mathbf{Z}_{c}^{\prime} \\ \vdots & \ddots & \vdots & \vdots \\ \mathbf{Z}_{\mathbf{N}} \mathbf{A} \sigma_{g_{1, N}} \mathbf{Z}_{1}^{\prime}+\mathbf{Z}_{\mathbf{N}} \mathbf{I} \sigma_{e_{1, N}} \mathbf{Z}_{1}^{\prime} & \cdots & \mathbf{Z}_{N} \mathbf{A} \sigma_{g_{N}}^{2} \mathbf{Z}_{N}^{\prime}+\mathbf{Z}_{N} \mathbf{I} \sigma_{e_{N}}^{2} \mathbf{Z}_{N}^{\prime} & \mathbf{Z}_{N} \mathbf{A} \sigma_{g_{N}, \beta} \mathbf{Z}_{c}^{\prime}+\mathbf{Z}_{N} \mathbf{I} \sigma_{e_{N}, \varepsilon} \mathbf{Z}_{c}^{\prime} \\ \mathbf{Z}_{c} \mathbf{A} \sigma_{g_{1}, \beta} \mathbf{Z}_{1}^{\prime}+\mathbf{Z}_{c} \mathbf{I} \sigma_{e_{1}, \varepsilon} \mathbf{Z}_{1}^{\prime} & \cdots & \mathbf{Z}_{c} \mathbf{A} \sigma_{g_{N}, \beta} \mathbf{Z}_{N}^{\prime}+\mathbf{Z}_{c} \mathbf{I} \sigma_{e_{N}, \varepsilon} \mathbf{Z}_{N}^{\prime} & \mathbf{Z}_{c} \mathbf{A} \sigma_{\beta}^{2} \mathbf{Z}_{c}^{\prime}+\mathbf{Z}_{c} \mathbf{I} \sigma_{\varepsilon}^{2} \mathbf{Z}_{c}^{\prime}\end{array}\right]$

where $\mathbf{Z}_{c}$ is an incidence matrix for the vector of the random genetic and residual effects, $\boldsymbol{\beta}$ and $\boldsymbol{\varepsilon}$, underlying $\mathbf{c}$. The genetic and residual variances of covariate $\mathbf{c}$ are denoted as $\sigma_{\beta}^{2}$ and $\sigma_{\varepsilon}^{2}$, respectively. The terms $\sigma_{g_{i}, \beta}$ and $\sigma_{e_{i}, \varepsilon}$ indicate the genetic and residual covariance between main trait and covariate at the $i$ th covariate levels $(i=$ $1, \ldots, N)$, respectively. The random genetic and residual effects of $y$ are the same as 
defined above. The random genetic and residual effect of $\mathbf{c}$ are assumed following a normal distribution with mean as zero and variance as $\mathbf{A} \sigma_{\beta}^{2}$ and $\mathbf{I} \sigma_{\varepsilon}^{2}$. The genetic (co)variance matrix of individual genetic effects in the multivariate model can be written as

$$
\mathbf{V}_{\mathbf{g}, \beta}=\left[\begin{array}{cc}
\boldsymbol{\Phi} \mathbf{K}_{\mathbf{y}} \boldsymbol{\Phi}^{\prime} & \boldsymbol{\Phi} \mathbf{K}_{\mathbf{y}, \mathbf{c}} \\
\mathbf{K}_{\mathbf{y}, \mathbf{c}}^{\prime} \boldsymbol{\Phi}^{\prime} & \operatorname{var}(\boldsymbol{\beta})
\end{array}\right]=\left[\begin{array}{cccc}
\sigma_{g_{1}}^{2} & \cdots & \sigma_{g_{1, N}} & \sigma_{g_{1}, \beta} \\
\vdots & \ddots & \vdots & \vdots \\
\sigma_{g_{N, 1}} & \cdots & \sigma_{g_{N}}^{2} & \sigma_{g_{N}, \beta} \\
\sigma_{g_{1}, \beta} & \cdots & \sigma_{g_{N}, \beta} & \sigma_{\beta}^{2}
\end{array}\right]
$$

where $\mathbf{K}_{\mathbf{y}}$ is the same as $\mathbf{K}$ defined above, and $\mathbf{K}_{\mathrm{y}, \mathbf{c}}$ consists of the covariance between $\boldsymbol{\beta}$ and the random regression coefficients, that is

$$
\mathbf{K}_{\mathbf{y}, \mathbf{c}}=\left[\begin{array}{c}
\operatorname{cov}\left(\boldsymbol{\alpha}_{0}, \boldsymbol{\beta}\right) \\
\operatorname{cov}\left(\boldsymbol{\alpha}_{1}, \boldsymbol{\beta}\right)
\end{array}\right] .
$$

The multivariate residual covariance structure is

$$
\mathbf{R}_{\mathbf{e}, \boldsymbol{\varepsilon}}=\left[\begin{array}{cc}
\operatorname{var}(\mathbf{e}) & \operatorname{cov}(\mathbf{e}, \boldsymbol{\varepsilon}) \\
\operatorname{cov}(\mathbf{e}, \boldsymbol{\varepsilon}) & \operatorname{var}(\boldsymbol{\varepsilon})
\end{array}\right],
$$

where $\mathbf{e}$ is the vector of residual effects for the main phenotypes, assuming that var (e) is homogenous across different levels of covariate values, i.e.,

$\sigma_{e_{1}}^{2}=\sigma_{e_{2}}^{2}=, \ldots,=\sigma_{e_{N}}^{2}$, which can be relaxed for the case of heterogeneous residual variances (see the next section), and $\boldsymbol{\varepsilon}$ is the vector of residual effects for the covariate, defined as above, and $\operatorname{var}(\boldsymbol{\varepsilon})$ is the residual variance of the covariate.

This model explicitly parameterises covariance between the random regression coefficients for the main phenotypes and the genetic effects underlying the covariate (i.e., $\mathbf{K}_{\mathbf{y , c}}$ ), therefore, is referred to as a genotype-covariate correlation and interaction (GCCI) model. Importantly, values for $\operatorname{cov}\left(\boldsymbol{\alpha}_{0}, \boldsymbol{\beta}\right)$ or $\operatorname{cov}(\mathbf{e}, \boldsymbol{\varepsilon})$ are often non-negligible. Neglecting these terms can cause confounding between G-C correlation and interaction, thereby generating spurious signals and biased estimates for the interaction. Yet many studies do not account for G-C correlations when estimating and testing $\mathrm{G}-\mathrm{C}$ interaction ${ }^{16}$.

MRNM accounting for heterogeneous residual variance (RCCI). The models we described so far assume that the residual variance for the main phenotypes, $\operatorname{var}(\mathbf{e})$, is homogeneous across different values of the covariate. However, it is often possible that residual-covariate $(\mathrm{R}-\mathrm{C})$ correlation and interaction exist, resulting in heterogeneous residual variances across different covariate values. To account for this possibility, MRNM can be further generalised as

$$
y_{i}=b_{i}+g_{i}+e_{i}=b_{i}+\alpha_{i 0}+\alpha_{i 1} \cdot c_{i}+\tau_{i 0}+\tau_{i 1} \cdot c_{i}
$$

where the residual term, $e_{i}$, can be also regressed on the covariate gradient, modelled with the zero and first order of random regression coefficients $\left(\tau_{i 0}\right.$ and $\tau_{i 1}$ ) and polynomial of the covariate.

The variance-covariance structure of the genetic effect for this model is the same as for the multivariate reaction norm model described in Eq. (2) in the previous section. The multivariate residual covariance structure in this generalised MRNM becomes

$$
\mathbf{R}_{\mathbf{e}, \boldsymbol{\varepsilon}}=\left[\begin{array}{cc}
\boldsymbol{\Phi M}_{\mathbf{y}} \boldsymbol{\Phi}^{\prime} & \boldsymbol{\Phi M}_{\mathbf{y}, \mathbf{c}} \\
\mathbf{M}_{\mathbf{y}, \mathbf{c}}^{\prime} \boldsymbol{\Phi}^{\prime} & \operatorname{var}(\boldsymbol{\varepsilon})
\end{array}\right]=\left[\begin{array}{cccc}
\sigma_{e_{1}}^{2} & \cdots & \sigma_{e_{1, N}} & \sigma_{e_{1}, \varepsilon} \\
\vdots & \ddots & \vdots & \vdots \\
\sigma_{e_{N, 1}} & \cdots & \sigma_{e_{N}}^{2} & \sigma_{e_{N}, \varepsilon} \\
\sigma_{e_{1}, \varepsilon} & \cdots & \sigma_{e_{N}, \varepsilon} & \sigma_{\varepsilon}^{2}
\end{array}\right]
$$

where $\mathbf{M}_{\mathbf{y}}$ is the variance and covariance matrix of random regression coefficients for the residual components and can be written as

$$
\mathbf{M}_{\mathbf{y}}=\operatorname{cov}\left(\boldsymbol{\tau}_{0}, \boldsymbol{\tau}_{1}\right)=\left[\begin{array}{cc}
\operatorname{var}\left(\boldsymbol{\tau}_{0}\right) & \operatorname{cov}\left(\boldsymbol{\tau}_{0}, \boldsymbol{\tau}_{1}\right) \\
\operatorname{cov}\left(\boldsymbol{\tau}_{0}, \boldsymbol{\tau}_{1}\right) & \operatorname{var}\left(\boldsymbol{\tau}_{1}\right)
\end{array}\right],
$$

where $\tau_{0}$ and $\tau_{1}$ are the zero and first order random regression coefficients for the residual effects. $\mathbf{M}_{\mathbf{y}, \mathbf{c}}$ is a vector with the covariance between $\boldsymbol{\varepsilon}$ and the random regression coefficients for the residual effects, and can be expressed as

$$
\mathbf{M}_{\mathbf{y}, \mathbf{c}}=\left[\begin{array}{c}
\operatorname{cov}\left(\boldsymbol{\tau}_{0}, \boldsymbol{\varepsilon}\right) \\
\operatorname{cov}\left(\boldsymbol{\tau}_{1}, \varepsilon\right)
\end{array}\right]
$$

RNM with multiple covariates. RNM can be further extended to include multiple covariates. A model fitting with multiple covariates can be expressed as

$$
y_{i}=b_{i}+\sum_{j=1}^{x} g_{i j}+e_{i}=b_{i}+\sum_{j=1}^{x}\left(\alpha_{i j 0}+\alpha_{i j 1} \cdot c_{i j}\right)+e_{i},
$$

where $x$ is the number of random effects, each of which is associated with a unique combination of a relationship matrix and covariate (see below), $\alpha_{i j 0}$ and $\alpha_{i j 1}$ are the zero and first order random regression coefficient for the $j$ th random effect and $c_{i j}$ is the covariate value for the $j$ th random effect. Therefore, this model is a multiple random effects model fitting multiple components ${ }^{22}$, but it allows the inclusion of interaction effects for each random effect. As in the original multiple random effects model, it is assumed that there is no correlation between the random effects $^{42}$.

The variance-covariance matrix of observed phenotypes $\left(y_{i}\right)$ for this multiple random effects model is

$\operatorname{var}(\mathbf{y})=\left[\begin{array}{ccc}\sum_{j=1}^{x} \mathbf{Z}_{1} \mathbf{A}_{j} \sigma_{\left(g_{1}\right)_{j}}^{2} \mathbf{Z}_{1}^{\prime}+\mathbf{Z}_{1} \mathbf{I} \sigma_{e_{1}}^{2} \mathbf{Z}_{1}^{\prime} & \cdots & \sum_{j=1}^{x} \mathbf{Z}_{1} \mathbf{A}_{j} \sigma_{\left(g_{1, N}\right)} \mathbf{Z}_{N}^{\prime}+\mathbf{Z}_{1} \mathbf{I} \sigma_{e_{1, N}} \mathbf{Z}_{N}^{\prime} \\ \vdots & \ddots & \vdots \\ \sum_{j=1}^{x} \mathbf{Z}_{\mathrm{N}} \mathbf{A}_{j} \sigma_{\left(g_{1, N}\right)_{j}} \mathbf{Z}_{1}^{\prime}+\mathbf{Z}_{\mathrm{N}} \mathbf{I} \sigma_{e_{1, N}} \mathbf{Z}_{1}^{\prime} & \cdots & \sum_{j=1}^{x} \mathbf{Z}_{N} \mathbf{A}_{j} \sigma_{\left(g_{N}\right)}^{2} \mathbf{Z}_{\mathrm{N}}^{\prime}+\mathbf{Z}_{N} \mathbf{I} \sigma_{e_{N}}^{2} \mathbf{Z}_{N}^{\prime}\end{array}\right]$,

where $\mathbf{A}_{\mathbf{j}}$ is the genomic relationship matrix for the $j$ th random effect, $\sigma_{\left(g_{i}\right)_{j}}^{2}$ is the genetic variance at the $i$ th covariate level for the $j$ th random effect, $\sigma_{\left(g_{1, N}\right)_{j}}$ is, for example, the genetic covariance between the first and the last covariate levels, and other terms are defined as above. As in the RNM fitting with a single covariate, $g_{i j}$ in each random effect $(j=1 \sim x)$ can be regressed on the covariate gradient in the same manner. The variance-covariance matrix of random regression coefficients for each random effect $\left(\mathbf{K}_{j}\right)$ can be written as

$$
\mathbf{K}_{j}=\operatorname{cov}\left(\boldsymbol{\alpha}_{j 0}, \boldsymbol{\alpha}_{j 1}\right)=\left[\begin{array}{cc}
\operatorname{var}\left(\boldsymbol{\alpha}_{j 0}\right) & \operatorname{cov}\left(\boldsymbol{\alpha}_{j 0}, \boldsymbol{\alpha}_{j 1}\right) \\
\operatorname{cov}\left(\boldsymbol{\alpha}_{j 0}, \boldsymbol{\alpha}_{j 1}\right) & \operatorname{var}\left(\boldsymbol{\alpha}_{j 1}\right)
\end{array}\right] .
$$

Similarly, the genetic (co)variance matrix of individual genetic effects between $N$ individuals can be obtained as

$$
\mathbf{V}_{\mathrm{g}_{j}}=\boldsymbol{\Phi}_{j} \mathbf{K}_{j} \boldsymbol{\Phi}_{j}^{\prime}=\left[\begin{array}{ccc}
\sigma_{\left(g_{1}\right)_{j}}^{2} & \cdots & \sigma_{\left(g_{1, N}\right)_{j}} \\
\vdots & \ddots & \vdots \\
\sigma_{\left(g_{1, N}\right)_{j}} & \cdots & \sigma_{\left(g_{N}\right)_{j}}^{2}
\end{array}\right],
$$

where $\boldsymbol{\Phi}_{j}$ is the $N \times 2$ matrix of covariate polynomials for the $j$ th random effect, and the variance-covariance components were defined as above. This multiple random effects model fitting with multiple covariates can be feasibly extended to MRNM with GCCI and RCCI although the number of parameters increases exponentially. All models described above can be fitted using MTG2 ${ }^{17}$.

Simulated data. Phenotypic simulation was based on individual genotypes from the GWAS data of the Atherosclerosis Risk in Communities Study (ARIC) cohort. We used autosomes only and applied the standard quality control (QC) to genotypes, which included MAF $>0.01$, SNP call rate $>0.95$, sample call rate $>0.95$ and Hardy-Weinberg Equilibrium $p$-value $>0.001$, keeping qualified genotyped SNPs. After the standard QC, 583,058 SNPs and 8,291 individuals remained. In addition, we estimated pair-wise relatedness from the remaining SNPs and randomly excluded one individual from each pair with an estimated relatedness greater than 0.05 . This relatedness cut-off QC reduced the sample to 7263 individuals.

Simulation under GCCI model (MRNM G-C model): We simulated phenotypes for the main response $(\mathbf{y})$ and covariate (c) under the GCCI model with the first order interaction effect, i.e., $k=1$. In the simulation, we used the following covariance structure for the $\mathbf{K}_{\mathbf{y}}$ matrix in Eq. (2) as

$$
\mathbf{K}_{\mathrm{y}}=\left[\begin{array}{cc}
\operatorname{var}\left(\boldsymbol{\alpha}_{0}\right) & \operatorname{cov}\left(\boldsymbol{\alpha}_{0}, \boldsymbol{\alpha}_{1}\right) \\
\operatorname{cov}\left(\boldsymbol{\alpha}_{0}, \boldsymbol{\alpha}_{1}\right) & \operatorname{var}\left(\boldsymbol{\alpha}_{1}\right)
\end{array}\right]=\left[\begin{array}{cc}
1 & 0.05 \\
0.05 & \operatorname{var}\left(\boldsymbol{\alpha}_{1}\right)
\end{array}\right] .
$$

We used a wide range of the G-C interaction with $\operatorname{var}\left(\boldsymbol{\alpha}_{1}\right)$ set at $0,0.25,0.5,0.75$ or 1 . For the covariate, $c_{i}=\mu_{i}+\beta_{i}+\varepsilon_{i}, \operatorname{var}(\boldsymbol{\beta})$ and $\operatorname{var}(\boldsymbol{\varepsilon})$ were set at 1 .

The values for the $\mathbf{K}_{\mathbf{y}, \mathbf{c}}$ matrix (Eq. 2) were used in the simulation as

$$
\mathbf{K}_{\mathbf{y}, \mathbf{c}}=\left[\begin{array}{c}
\operatorname{cov}\left(\boldsymbol{\alpha}_{0}, \boldsymbol{\beta}\right) \\
\operatorname{cov}\left(\boldsymbol{\alpha}_{1}, \boldsymbol{\beta}\right)
\end{array}\right]=\left[\begin{array}{c}
0.5 \\
0
\end{array}\right]
$$

and

$$
\mathbf{R}_{\mathbf{e}, \boldsymbol{\varepsilon}}=\left[\begin{array}{cc}
\operatorname{var}(\mathbf{e}) & \operatorname{cov}(\mathbf{e}, \boldsymbol{\varepsilon}) \\
\operatorname{cov}(\mathbf{e}, \boldsymbol{\varepsilon}) & \operatorname{var}(\boldsymbol{\varepsilon})
\end{array}\right]=\left[\begin{array}{cc}
1 & 0.3 \\
03 & 1
\end{array}\right] .
$$

Simulation under RCCI model (MRNM R-C model): In this simulation, we used the following covariance structure for the $\mathbf{K}_{\mathrm{y}}$ matrix in Eq. (2) as

$$
\mathbf{K}_{\mathbf{y}}=\left[\begin{array}{cc}
\operatorname{var}\left(\boldsymbol{\alpha}_{0}\right) & \operatorname{cov}\left(\boldsymbol{\alpha}_{0}, \boldsymbol{\alpha}_{1}\right) \\
\operatorname{cov}\left(\boldsymbol{\alpha}_{0}, \boldsymbol{\alpha}_{1}\right) & \operatorname{var}\left(\boldsymbol{\alpha}_{1}\right)
\end{array}\right]=\left[\begin{array}{ll}
1 & 0 \\
0 & 0
\end{array}\right] .
$$

For the covariate, $c_{i}=\mu_{i}+\beta_{i}+\varepsilon_{i}, \operatorname{var}(\boldsymbol{\beta})$ and $\operatorname{var}(\boldsymbol{\varepsilon})$ were set at 1 . The values for the $\mathbf{K}_{\mathbf{y}, \mathbf{c}}$ matrix (Eq. 2) were used in the simulation as

$$
\mathbf{K}_{\mathrm{y}, \mathrm{c}}=\left[\begin{array}{c}
\operatorname{cov}\left(\boldsymbol{\alpha}_{0}, \boldsymbol{\beta}\right) \\
\operatorname{cov}\left(\boldsymbol{\alpha}_{1}, \boldsymbol{\beta}\right)
\end{array}\right]=\left[\begin{array}{c}
0.5 \\
0
\end{array}\right] .
$$

The $\mathbf{M}_{\mathbf{y}}$ and $\mathbf{M}_{\mathbf{y}, \mathbf{c}}$ matrices (Eqs 3 and 4) were specified in the simulation as follows:

$\mathbf{M}_{\mathbf{y}}=\left[\begin{array}{cc}\operatorname{var}\left(\boldsymbol{\tau}_{0}\right) & \operatorname{cov}\left(\boldsymbol{\tau}_{0}, \boldsymbol{\tau}_{1}\right) \\ \operatorname{cov}\left(\boldsymbol{\tau}_{0}, \boldsymbol{\tau}_{1}\right) & \operatorname{var}\left(\boldsymbol{\tau}_{1}\right)\end{array}\right]=\left[\begin{array}{cc}1 & 0.05 \\ 0.05 & \operatorname{var}\left(\boldsymbol{\tau}_{1}\right)\end{array}\right]$ with $\operatorname{var}\left(\boldsymbol{\tau}_{1}\right)=0,0.25$ or 1 
and

$$
\mathbf{M}_{\mathbf{y}, \mathbf{c}}=\left[\begin{array}{c}
\operatorname{cov}\left(\boldsymbol{\tau}_{0}, \boldsymbol{\varepsilon}\right) \\
\operatorname{cov}\left(\boldsymbol{\tau}_{1}, \boldsymbol{\varepsilon}\right)
\end{array}\right]=\left[\begin{array}{c}
\operatorname{cov}\left(\boldsymbol{\tau}_{0}, \boldsymbol{\varepsilon}\right) \\
0
\end{array}\right] \text { with } \operatorname{cov}\left(\boldsymbol{\tau}_{0}, \boldsymbol{\varepsilon}\right)=0 \text { or } 0.3 .
$$

Simulation under GCCI and RCCI model (MRNM Full model): Similar to the GCCI simulations above, we used values for the $\mathbf{K}_{\mathbf{y}}$ matrix in Eq. (2) as

$$
\mathbf{K}_{\mathbf{y}}=\left[\begin{array}{cc}
\operatorname{var}\left(\boldsymbol{\alpha}_{0}\right) & \operatorname{cov}\left(\boldsymbol{\alpha}_{0}, \boldsymbol{\alpha}_{1}\right) \\
\operatorname{cov}\left(\boldsymbol{\alpha}_{0}, \boldsymbol{\alpha}_{1}\right) & \operatorname{var}\left(\boldsymbol{\alpha}_{1}\right)
\end{array}\right]=\left[\begin{array}{cc}
1 & 0.05 \\
0.05 & \operatorname{var}\left(\boldsymbol{\alpha}_{1}\right)
\end{array}\right] .
$$

We performed simulations with $\operatorname{var}\left(\boldsymbol{\alpha}_{1}\right)$ set at $0,0.25$ or 1 , and for the covariate, both $\operatorname{var}(\boldsymbol{\beta})$ and $\operatorname{var}(\boldsymbol{\varepsilon})$ were set at 1 .

The $\mathbf{K}_{\mathbf{y}, \mathfrak{c}}, \mathbf{M}_{\mathbf{y}}$ and $\mathbf{M}_{\mathbf{y}, \mathbf{c}}$ matrices (Eqs. 3 and 4) were specified in the simulation as follows:

$$
\begin{gathered}
\mathbf{K}_{\mathbf{y}, \mathbf{c}}=\left[\begin{array}{c}
\operatorname{cov}\left(\boldsymbol{\alpha}_{0}, \boldsymbol{\beta}\right) \\
\operatorname{cov}\left(\boldsymbol{\alpha}_{1}, \boldsymbol{\beta}\right)
\end{array}\right]=\left[\begin{array}{c}
\operatorname{cov}\left(\boldsymbol{\alpha}_{0}, \boldsymbol{\beta}\right) \\
0
\end{array}\right] \text { with } \operatorname{cov}\left(\boldsymbol{\alpha}_{0}, \boldsymbol{\beta}\right)=0 \text { or } 0.5, \\
\mathbf{M}_{\mathbf{y}}=\left[\begin{array}{cc}
\operatorname{var}\left(\boldsymbol{\tau}_{0}\right) & \operatorname{cov}\left(\boldsymbol{\tau}_{0}, \boldsymbol{\tau}_{1}\right) \\
\operatorname{cov}\left(\boldsymbol{\tau}_{0}, \boldsymbol{\tau}_{1}\right) & \operatorname{var}\left(\boldsymbol{\tau}_{1}\right)
\end{array}\right]=\left[\begin{array}{cc}
1 & 0.05 \\
0.05 & \operatorname{var}\left(\boldsymbol{\tau}_{1}\right)
\end{array}\right] \text { with } \operatorname{var}\left(\boldsymbol{\tau}_{1}\right)=0.25 \text { or } 1
\end{gathered}
$$

and

$$
\mathbf{M}_{\mathbf{y}, \mathbf{c}}=\left[\begin{array}{l}
\operatorname{cov}\left(\boldsymbol{\tau}_{0}, \boldsymbol{\varepsilon}\right) \\
\operatorname{cov}\left(\boldsymbol{\tau}_{1}, \boldsymbol{\varepsilon}\right)
\end{array}\right]=\left[\begin{array}{c}
\operatorname{cov}\left(\boldsymbol{\tau}_{0}, \boldsymbol{\varepsilon}\right) \\
0
\end{array}\right] \text { with } \operatorname{cov}\left(\boldsymbol{\tau}_{0}, \boldsymbol{\varepsilon}\right)=0 \text { or } 0.3
$$

According to the normal distribution assumption in the models, all genetic and residual values were drawn from normal distributions with mean zero and variance-covariance structures specified as above. In the presence or absence of interactions with simulated data under these various models, we assessed bias, type I error rate and power for LDSC $^{19,20}$, GREML ${ }^{18}$, MVGREML ${ }^{17}$, RR-GREML $^{16}$, GCI-GREML ${ }^{18}$, RNM, and MRNM. We used likelihood ratio tests to get $p$-values to detect interaction effects and also estimated variance components in the models. We used inverse normal $p$-values transformed from the raw $p$-values using qnorm function in $\mathrm{R}^{43}$ for a clearer comparison across the methods.

Real data. Data and Quality control: We used the UK Biobank data ${ }^{24}$, which initially contained 488,377 individuals and 92,693,895 imputed SNPs across autosomes. Stringent quality control was applied to the genotype data at both individual and SNP levels. Specifically, we excluded individuals who met one of the following criteria: (1) does not have white British ancestry, (2) has a genotype missing rate $>0.05$, (3) whose reported gender does not match with the gender inferred using genotype data, and (4) has a putative sex chromosome aneuploidy. At the SNP level, we excluded SNPs with an INFO score $<0.6$, with a $\mathrm{MAF}<0.01$, with a Hardy-Weinberg equilibrium $p$-value $<1 \mathrm{E}-4$, or with a call rate $<0.95$. We excluded ambiguous or duplicated SNPs. We only used the HapMap 3 SNPs in the main analyses because they are reliable and robust to bias in estimating SNP-based heritability and genetic correlation ${ }^{44-46}$. In addition, we excluded individual population outliers with the first or second PC outside six standard deviations of the population mean. For individuals who were in the first and second releases of UK biobank genotype data, we calculated the discordance rate between imputed genotype of the two versions for each individual and for each SNP, and excluded individuals and SNPs with a discordance rate lager than 0.05 . We also excluded one individual randomly from any pair with a genomic relationship larger than 0.05 . After the QC above, 288,866 individuals and $1,130,918$ SNPs remained. Of these remaining individuals 91,472 were from the first release of UK Biobank (denoted as UKBB1) and 66,281 individuals with complete records of covariates were used in the main analyses. The rest of 197,394 individuals were from the second release of UK Biobank (denoted as UKBB2) and 115,053 with complete records of covariates were used in the validation and metaanalyses.

Main response variable and covariates: We applied the novel (M)RNM model using BMI as the main response variable to estimate the GCCI/RCCI components with each of several covariates, including pack years of smoking (SMK), neuroticism score (NEU) or the first principal component (PC1) provided by the UK Biobank. We also fitted the model that includes multiple covariates (e.g., SMK and NEU) jointly, i.e., RNM with multiple covariates. For all analyses, covariates were standardised as mean zero and variance 1. Prior to model fitting, we adjusted the main response variable (BMI) for confounders including genotype batch, assessment centre at which participant consented, year of birth, sex, age, diet variation, diet change, the first 15 PCs, SMK, weekly alcohol consumption (ALC) and Townsend deprivation index at recruitment (TDI). In the analyses using NEU and $\mathrm{PC} 1$ as the covariates, we further adjusted BMI for NEU to correct the mean difference. The distribution of each covariate is in Supplementary Fig. 16.

When including the covariate (i.e., SMK, NEU, or PC1) as the second trait in a MRNM, it was also pre-adjusted for the confounders in a similar way as for the main trait (i.e., BMI). For instance, as the second trait in a MRNM, SMK was preadjusted for BMI, genotype batch, assessment centre at which participant consented, year of birth, sex, age, diet variation, diet change, the first 15 PCs, ALC, and TDI. NEU was pre-adjusted for BMI, genotype batch, assessment centre at which participant consented, year of birth, sex, age, diet variation, diet change, the first 15 PCs, ALC, TDI and SMK. PC1 was pre-adjusted for BMI, genotype batch, assessment centre at which participant consented, year of birth, sex, age, diet variation, diet change, the first 15 PCs except the first one (PC1), ALC, TDI, SMK and NEU.

Detailed information regarding covariates used in the interaction models is described below and that for other confounders used to adjust the main phenotypes is in Supplementary Note 6.

Pack years of smoking (SMK): We combined pack years adult smoking as proportion of life span exposed to smoking (UK Biobank data field: 20162) and ever smoked (UK Biobank data field 20160) as SMK. The distribution of SMK is in Supplementary Fig. 16. For RR-GREML and GCI-GREML, following Robinson et al. ${ }^{16}$, we stratified SMK into four levels: 8,773 individuals with SMK $>0.8,9,192$ individuals with $0.5 \leq \mathrm{SMK} \leq 0.8,11,741$ individuals with $0<\mathrm{SMK}<0.5$, and 36,575 individuals with $\mathrm{SMK}=0$ (i.e., never smoked).

Neuroticism score (NEU): The neuroticism score (data field 20127) of a given individual was indexed by the number of 'yes's to 12 touchscreen questions that evaluate neurotic behaviours. The distribution of NEU is in Supplementary Fig. 16. For RR-GREML and GCI-GREML, we stratified the data into four groups according to NEU level: 20,901 individuals with $\mathrm{NEU} \leq 2,16,161$ individuals with $2<\mathrm{NEU} \leq 5,10,895$ individuals with $5<\mathrm{NEU} \leq 8$, and 6,417 individuals with $8<$ $\mathrm{NEU} \leq 12$.

The fist principal components of genotype (PC1): PCs were pre-calculated by the UK Biobank. Detailed information regarding the calculation is described elsewhere ${ }^{47}$. Briefly, PCs-loadings were estimated using fastPCA ${ }^{48}$ based on 407,219 unrelated individuals and 147,604 markers that were pruned to minimise linkage disequilibrium, onto where all samples were projected, to generate a set of PC scores. For RR-GREML and GCI-GREML, we stratified the sample into four groups based on quartiles of PC1.

Meta analyses of real data: The proposed MRNM requires individual-level genotype data, which makes it computationally demanding. As sample size increases (e.g., the second release of UK Biobank), the computing time increases substantially. To complete the analyses within a reasonable timeframe, we used a meta-analysis approach. We performed two sets of meta-analyses, one across two groups within UKBB1 to assess the performance of the meta-analysis, compared to that of the whole UKBB1 data analysis, and the other across UKBB1 and UKBB2.

Meta-analyses within UKBB1. We randomly divided the UKBB1 into two groups of equal size (denoted as g1 and g2), and fitted all models mentioned above for each group. $P$-values from each group were meta-analysed using the Fisher's method $^{31}$. We then compared these $p$-values with those based on the whole UKBB1 data set.

Meta-analyses across UKBB1 and UKBB2. In UKBB2, 197,394 individuals with genotype data passed the QC, of which $94 \mathrm{~K}$ have no missing covariates and main response. Similar to meta-analyses within UKBB1, we randomly divided the UKBB2 into two groups of equal size (denoted as G1 and G2), and fitted all models mentioned above for each group. We then meta-analysed the results from G1, G2, and UKBB1 (denoted as G0) using the Fisher's method ${ }^{31}$. For UKBB2, the same pre-adjustment as for UKBB1 was applied to the main response and covariates as the second trait in MRNM.

URLs. UK Biobank, http://www.ukbiobank.ac.uk/ LDSC, https://github.com/bulik/ldsc

GCTA, http://cnsgenomics.com/software/gcta/ Plink1.9, https://www.cog-genomics.org/plink2 MTG2, https://sites.google.com/site/honglee0707/mtg2

Reporting summary. Further information on research design is available in the Nature Research Reporting Summary linked to this article.

\section{Data Availability}

The simulated data can be obtained from the authors on request. We also used the genotype data of ARIC study under accession code phs000090 in the database of Genotypes and Phenotypes. All other relevant data is available upon request. The source data underlying Figs. 1-8 and Supplementary Figs. 1-23 are provided as a Source Data file.

\section{Code availability}

The RNM and MRNM for GCCI and RCCI analyses are fully implemented in MTG2 version 2.14 .

Received: 12 December 2018 Accepted: 18 April 2019

Published online: 20 May 2019

\section{References}

1. Manuck, S. B. \& McCaffery, J. M. Gene-Environment Interaction. Annu. Rev. Psychol. 65, 41-70 (2014). 
2. Moore, R. et al. A linear mixed model approach to study multivariate geneenvironment interactions. bioRxiv, 270611 (2018).

3. Dahl, A., Cai, N., Flint, J. \& Zaitlen, N. GxEMM: extending linear mixed models to general gene-environment interactions. bioRxiv, 397638 (2018).

4. Wang, T. et al. Pleiotropy of genetic variants on obesity and smoking phenotypes: results from the Oncoarray Project of The International Lung Cancer Consortium. PLoS ONE 12, e0185660 (2017).

5. Canoy, D. et al. Cigarette smoking and fat distribution in 21,828 British men and women: a population-based study. Obesity 13, 1466-1475 (2005).

6. Chiolero, A., Faeh, D., Paccaud, F. \& Cornuz, J. Consequences of smoking for body weight, body fat distribution, and insulin resistance. Am. J. Clin. Nutr. 87, 801-809 (2008).

7. Carreras-Torres, R. et al. Role of obesity in smoking behaviour: Mendelian randomisation study in UK Biobank. BMJ 361, k1767 (2018).

8. Feinberg, A. P. Phenotypic plasticity and the epigenetics of human disease. Nature 447, 433-440 (2007).

9. Fusco, G. \& Minelli, A. Phenotypic plasticity in development and evolution: facts and concepts. Philos. Trans. R. Soc. Lond. B Biol. Sci. 365, 547-556 (2010).

10. West-Eberhard, M. J. Phenotypic plasticity and the origins of diversity. Annu. Rev. Ecol. Syst. 20, 249-278 (1989)

11. Purcell, S. Variance components models for gene-environment interaction in twin analysis. Twin Res. Hum. Genet. 5, 554-571 (2002).

12. Caspi, A., Hariri, A. R., Holmes, A., Uher, R. \& Moffitt, T. E. Genetic sensitivity to the environment: the case of the serotonin transporter gene and its implications for studying complex diseases and traits. Focus 8, 398-416 (2010).

13. Nussey, D. H., Wilson, A. J. \& Brommer, J. E. The evolutionary ecology of individual phenotypic plasticity in wild populations. J. Evol. Biol. 20, 831-844 (2007).

14. Gregorius, H. R. \& Namkoong, G. Joint analysis of genotypic and environmental effects. Theor. Appl. Genet. 72, 413-422 (1986).

15. Jarquín, D. et al. A reaction norm model for genomic selection using highdimensional genomic and environmental data. Theor. Appl. Genet. 127, 595-607 (2014).

16. Robinson, M. R. et al. Genotype-covariate interaction effects and the heritability of adult body mass index. Nat. Genet. 49, 1174 (2017).

17. Lee, S. H. \& van der Werf, J. MTG2: An efficient algorithm for multivariate linear mixed model analysis based on genomic information. Bioinformatics 32, 1420-1422 (2016).

18. Yang, J., Lee, S. H., Goddard, M. E. \& Visscher, P. M. GCTA: A tool for genome-wide complex trait analysis. Am. J. Hum. Genet. 88, 76-82 (2011).

19. Bulik-Sullivan, B. et al. An Atlas of genetic correlations across human diseases and traits. Nat. Genet. 47, 1236-1241 (2015).

20. Bulik-Sullivan, B. K. et al. LD Score regression distinguishes confounding from polygenicity in genome-wide association studies. Nat. Genet. $\mathbf{4 7}$, 291-295 (2015).

21. Finucane, H. K. et al. Partitioning heritability by functional annotation using genome-wide association summary statistics. Nat. Genet. 47, 1228-1235 (2015).

22. Maier, R. et al. Joint analysis of psychiatric disorders increases accuracy of risk prediction for schizophrenia, bipolar disorder, and major depressive disorder. Am. J. Hum. Genet. 96, 283-294 (2015).

23. Ge, T., Chen, C.-Y., Neale, B. M., Sabuncu, M. R. \& Smoller, J. W. Phenomewide heritability analysis of the UK Biobank. PLoS Genet. 13, e1006711 (2017).

24. Collins, R. What makes UK Biobank special? Lancet 379, 1173-1174 (2012).

25. Heaven, P. C. L., Mulligan, K., Merrilees, R., Woods, T. \& Fairooz, Y. Neuroticism and conscientiousness as predictors of emotional, external, and restrained eating behaviors. Int. J. Eat. Disord. 30, 161-166 (2001).

26. Sutin, A. R. \& Terracciano, A. Personality traits and body mass index: Modifiers and mechanisms. Psychol. Health 31, 259-275 (2016).

27. Tropf, F. C. et al. Hidden heritability due to heterogeneity across seven populations. Nat. Hum. Behav. 1, 757 (2017).

28. The Brainstorm Consortium. Analysis of shared heritability in common disorders of the brain. Science 360, eaap8757 (2018).

29. Duncan, L. et al. Significant locus and metabolic genetic correlations revealed in genome-wide association study of anorexia nervosa. Am. J. Psychiatry 174, 850-858 (2017).

30. Ni, G. et al. Estimation of genetic correlation via linkage disequilibrium score regression and genomic restricted maximum likelihood. Am. J. Hum. Genet. 102, 1185-1194 (2018).

31. Evangelou, E. \& Ioannidis, J. P. A. Meta-analysis methods for genomewide association studies and beyond. Nat. Rev. Genet. 14, 379-389 (2013).

32. Ritz, B. R. et al. Lessons learned from past gene-environment interaction successes. Am. J. Epidemiol. 186, 778-786 (2017).
33. Nordestgaard, B. G. et al. The effect of elevated body mass index on ischemic heart disease risk: causal estimates from a Mendelian randomisation approach. PLoS Med. 9, e1001212 (2012).

34. Gilmour, A. R., Gogel, B. J., Cullis, B. R. \& Thompson, R. ASReml User Guide 3.0. (VSN International Ltd, Hemel Hempstead, UK, 2009).

35. Meyer, K. Estimating covariance functions for longitudinal data using a random regression model. Genet. Sel. Evol. 30, 221 (1998).

36. Kirkpatrick, M., Lofsvold, D. \& Bulmer, M. Analysis of the inheritance, selection and evolution of growth trajectories. Genetics 124, 979-993 (1990).

37. Pigliucci, M. Evolution of phenotypic plasticity: where are we going now? Trends Ecol. Evol. 20, 481-486 (2005).

38. Martin, J. G. A., Nussey, D. H., Wilson, A. J. \& Reale, D. Measuring individual differences in reaction norms in field and experimental studies: a power analysis of random regression models. Methods Ecol. Evol. 2, 362-374 (2011).

39. Cole, S. R. et al. Illustrating bias due to conditioning on a collider. Int. J. Epidemiol. 39, 417-420 (2009).

40. Munafò, M. R., Tilling, K., Taylor, A. E., Evans, D. M. \& Davey Smith, G. Collider scope: when selection bias can substantially influence observed associations. Int. J. Epidemiol. 47, 226-235 (2017).

41. Young, A. I., Wauthier, F. L. \& Donnelly, P. Identifying loci affecting trait variability and detecting interactions in genome-wide association studies. Nat. Genet. 50, 1608-1614 (2018).

42. Lynch, M. \& Walsh, B. Genetics and analysis of quantitative traits, (Sinauer Sunderland, MA 1998).

43. R Development Core Team. R: A language and environment for statistical computing (2013).

44. Cross-Disorder Group of the Psychiatric Genomics Consortium. Genetic relationship between five psychiatric disorders estimated from genome-wide SNPs. Nat. Genet. 45, 984-994 (2013).

45. Ripke, S. et al. Genome-wide association analysis identifies 13 new risk loci for schizophrenia. Nat. Genet. 45, 1-26 (2013).

46. Lee, S. H. et al. Estimation of SNP heritability from dense genotype data. Am. J. Hum. Genet. 93, 1151-1155 (2013)

47. Bycroft, C. et al. Genome-wide genetic data on $~ 500,000$ UK Biobank participants. bioRxiv, 166298 (2017).

48. Galinsky, K. J. et al. Fast principal-component analysis reveals convergent evolution of ADH1B in Europe and East Asia. Am. J. Hum. Genet. 98, 456-472 (2016).

\section{Acknowledgements}

This research is supported by the Australian National Health and Medical Research Council (1080157, 1087889, 1078901), and the Australian Research Council (DP160102126, FT160100229). This research has been conducted using the UK Biobank Resource. UK Biobank (http://www.ukbiobank.ac.uk) Research Ethics Committee (REC) approval number is $11 / \mathrm{NW} / 0382$. Our reference number approved by UK Biobank is 14575 . The Atherosclerosis Risk in Communities Study is carried out as a collaborative study supported by National Heart, Lung, and Blood Institute contracts (HHSN268201100005C, HHSN268201100006C, HHSN268201100007C, HHSN268201100008C, HHSN268201100009C, HHSN268201100010C, HHSN268201100011C, and HHSN268201100012C). We thank the staff and participants of the ARIC study for their important contributions. Funding for GENEVA was provided by National Human Genome Research Institute grant U01HG004402 (E. Boerwinkle).

\section{Author contributions}

S.H.L. conceived the idea and directed the study. G.N. and S.H.L. performed the analyses. N.R.W., J.v.d.W. and E.H. provided critical feedback and key elements in interpreting the results. S.H.L., G.N., N.R.W., J.v.d.W., E.H. and X.Z. drafted the manuscript. All authors contributed to editing and approval of the final manuscript.

\section{Additional information}

Supplementary Information accompanies this paper at https://doi.org/10.1038/s41467019-10128-w.

\section{Competing interests: The authors declare no competing interests.}

Reprints and permission information is available online at http://npg.nature.com/ reprintsandpermissions/

Journal peer review information: Nature Communications thanks Alexander Young and other anonymous reviewer(s) for their contribution to the peer review of this work. Peer reviewer reports are available.

Publisher's note: Springer Nature remains neutral with regard to jurisdictional claims in published maps and institutional affiliations. 
(c) (i) Open Access This article is licensed under a Creative Commons Attribution 4.0 International License, which permits use, sharing, adaptation, distribution and reproduction in any medium or format, as long as you give appropriate credit to the original author(s) and the source, provide a link to the Creative Commons license, and indicate if changes were made. The images or other third party material in this article are included in the article's Creative Commons license, unless indicated otherwise in a credit line to the material. If material is not included in the article's Creative Commons license and your intended use is not permitted by statutory regulation or exceeds the permitted use, you will need to obtain permission directly from the copyright holder. To view a copy of this license, visit http://creativecommons.org/ licenses/by/4.0/.

(C) The Author(s) 2019 